\title{
On the spectral and conservation properties of nonlinear discretization operators.
}

\author{
D. Fauconnier, E. Dick \\ Department of Flow, Heat and Combustion Mechanics, Ghent University, \\ St. Pietersnieuwstraat 41, B-9000 Ghent, Belgium
}

\begin{abstract}
Following the study of Pirozzoli [1], the objective of the present work is to provide a detailed theoretical analysis of the spectral properties and the conservation properties of nonlinear finite difference discretizations. First, a Nonlinear Spectral Analysis (NSA) is proposed in order to study the statistical behaviour of the modified wavenumber of a nonlinear finite difference operator, for a large set of synthetic scalar fields with prescribed energy spectrum and random phase. Second, the necessary conditions for local and global conservation of momentum and kinetic energy are derived and verified for nonlinear discretizations. Because the nonlinear mechanisms result in a violation of the energy conservation conditions, the NSA is used to quantify the energy imbalance. Third, the effect of aliasing errors due to the nonlinearity is analyzed. Finally, the theoretical observations are verified for two simple, thought relevant, numerical simulations.
\end{abstract}

Keywords: Nonlinear finite difference schemes, spectral properties, modified wavenumber, Nonlinear Spectral Analysis, conservation properties.

\section{Introduction}

In Computational Fluid Dynamics (CFD) and Computational Aero-Acoustics (CAA), the use of nonlinear discretizations for the spatial derivative operators in the differential equations, is widespread. Although these nonlinear discretization operators come in various forms and may serve different purposes, they have a nonlinear mechanism in common that results in the application of different discretization stencils in different nodes throughout the computational domain. The particular selection of the discretization stencil in a node of interest, is typically based on a smoothness-criterion of the solution in that node and surrounding nodes.

The oldest, and best known nonlinear discretization scheme is the upwind scheme, proposed by Courant et al. [2] in 1952. The upwind strategy involves a discretization stencil which is biased in the direction determined by the sign of the transporting velocity, i.e. the upwind direction. As long as the Courant-Friederichs-Lewy condition is satisfied, the scheme remains stable due to the intrinsic numerical dissipation of the eccentric stencils. Although this intrinsic stability is the most important asset of upwind schemes, the excessive dissipation is often considered undesirable for accurate numerical simulation of e.g. turbulent flows or acoustic wave propagation. Since the sign of the transporting velocity may alternate in the computational domain, the discretization stencil will alternate with it, making the upwind scheme de facto nonlinear.

Another class of nonlinear schemes, introduced in the early 80's by Harten [3], are the Total Variation Diminishing (TVD) schemes. These TVD schemes involve flux or slope limiters which must ensure the monotonicity of the solution at all times. If the solution is sufficiently smooth in a node of interest, a $2^{\text {nd }}$-order central discretization stencil is selected, which intrinsically lacks numerical dissipation. However, if the solution is not smooth on the computational grid, displaying spurious oscillations (e.g. Gibbs phenomenon, odd-even decoupling, etc.), steep gradients or discontinuities (e.g. shocks, etc.), an appropriate $1^{s t}$ - or $2^{\text {nd }}$-order upwind discretization is enforced to ensure the monotonicity of the solution. Although this makes TVD schemes highly stable, these methods have the disadvantage

Email address: dieter.fauconnier@ugent.be (D. Fauconnier) 
that the $1^{\text {st }}$-order upwind scheme is also engaged near smooth extrema, leading to unnecessary dissipation [4].

In order to obtain a higher order of accuracy, Harten et al. [5] introduced the Essentially Non-Oscillatory (ENO) discretization schemes. Here, the discretization stencils in any node of interest are constructed by selecting the "smoothest" interpolating polynomial of the solution, from a hierarchy of candidate interpolating polynomials with varying stencil support. Once the smoothest interpolant is known, a smooth approximation of the derivative is obtained readily. Later, Weighted Essentially Non-Oscillatory (WENO) schemes were developed, which use a convex combination of all candidate interpolating polynomials in the defined hierarchy [4]. Since the discretization stencils of both ENO and WENO schemes can differ from node to node, they are nonlinear by definition.

Recently Fauconnier et al. [6] developed a class of Dynamic Finite Difference (DFD) scheme. In contrast to the previously discussed schemes, the objective is not to guard the smoothness of the solution, but to obtain higher accuracy by minimizing the dispersion error of the central discretization stencil. This is achieved by reformulating the basic central discretization stencil as a function of an adjustable coefficient, which is then determined by a least square minimization on two grid resolutions. If the entire domain is taken into account in the minimization procedure, one obtains the same optimized discretization stencil in each node of the domain. However, if only the vicinity of the node of interest is taken as minimization region, then the optimized stencil varies from node to node, leading to a nonlinear discretization throughout the domain. We refer to $[6,7]$ for further information.

Despite the fact that nonlinear schemes are widely used in CFD, e.g. in applications concerning compressible flows, shock capturing, shock-turbulence interaction and wave propagation, their nonlinear behaviour and its impact on the solution, has been discussed in only a few studies. In 2001, Ladeinde et al. [8] compared the resolution of the small scale structures in the high-wavenumber region of the turbulent compressible energy spectrum, for three finite difference schemes: a linear compact scheme, an ENO scheme and a WENO scheme. They noticed that the nonlinear stencil adaptation of the ENO and WENO schemes leads to the generation of numerical small-scale turbulence, polluting the tail of the energy spectrum and limiting the useful resolution range of these schemes. Removing these spurious modes, improved the results. In 2006, Pirozzoli [1] investigated in more detail the spectral properties of some shockcapturing schemes such as TVD, ENO and WENO. The author motivated that "even though seldom stated explicitly, numerical tests show that the genuinely nonlinear mechanisms underlying shock-capturing schemes have a dramatic impact upon the computed solution, and their actual behavior may be very different from the one predicted on purely linear grounds." In order to study some effects of the nonlinearity on a solution in wavenumber space, an Approximate Dispersion Relation for nonlinear schemes was used, which relied on the extraction of a modified wavenumber from the convection of a sinusoidal wave with a certain wavenumber over a small distance. However, some caveats were reported in [1]. Indeed, this quasi-linear method did not consider solutions that contain an entire spectrum of scales. Further, the impact of the spurious modes, generated by the nonlinear schemes, and other nonlinear interactions on the evolution of a transported field is not considered. Based on this work, Bogey et al. [9] argued that "in order to assess the quality of the solutions, in particular in aeroacoustic studies, there is an urgent need of analysing the spectral properties of the shock-capturing schemes in the Fourier space". In Fauconnier et al. [6], the spectral behaviour of the nonlinear DFD scheme was investigated. The authors showed analytically that the modified wavenumber of the nonlinear scheme equals that of the $6^{\text {th }}$-order tridiagonal compact scheme for a single wave solution. A "dual-wave analysis" was presented in which the transfer functions of both schemes were calculated for a field with two independent wave components with equal amplitudes. A small loss of accuracy was noticed, due to the nonlinear interactions of the two waves. This was confirmed by the numerical study on the viscous Burgers' equation. They concluded that the nonlinear mechanism produces spurious scales in the entire wavenumber range, severely polluting the solution and substantially reducing the accuracy of the Burgers' solution. This is in accordance with the work of Ladeinde et al. [8].

Following the motivation of Pirozzoli [1] and Bogey et al. [9], the present work is an attempt to further analyze in detail, the spectral properties, as well as conservation properties, for a representative selection of nonlinear schemes. Moreover, the impact of the nonlinearity on the solution of a few elementary problems is investigated. First we analyze the spectral properties of a selection of nonlinear schemes, for a synthetic field containing a spectrum of Fourier modes up to a certain cutoff. The modes' amplitudes satisfy a certain scaling law, whereas the modes' random phases are uniformly distributed. This gives insight about the nonlinear interactions and their impact on the modified wavenumber and the energy spectrum. Second, we investigate analytically the conservation properties of nonlinear schemes. We verify whether the nonlinear schemes satisfy the necessary conditions for the conservation of momentum and kinetic energy in the simple, though essential and relevant, case of a one-dimensional linear convection equation. 
We show that nonlinear schemes can be formulated in order to satisfy the requirements for momentum conservation. However, we prove analytically that due to the nonlinear mechanism, the conditions for the conservation of kinetic energy can never be satisfied. Since nonlinear schemes violate the conditions for conservation of kinetic energy, we also investigate the impact of the nonlinearity on the energy balance, by means of a nonlinear spectral analysis. A third topic, related to the previous one, concerns the occurrences of aliasing errors due to the nonlinear interactions. More specifically, we analyze the impact of aliasing on the energy balance. We emphasize that the nonlinear spectral analysis, used to assess the impact of nonlinearity on various properties, is only valid for a single evaluation (i.e. a single iteration) of the nonlinear discretization scheme for a predefined scalar field, and does not take into account nonlinear influences due to repeated evaluation (i.e. multiple iterations). Finally, we verify our findings in two fundamental simulations, i.e. the linear convection equation and a viscous Burgers' equation with energy-conserving forcing.

\section{A selection of Spatially Nonlinear Finite Difference Schemes}

Consider the one-dimensional hyperbolic conservation law for a scalar field $u(x, t), x \in[0, L]$,

$$
\frac{\partial u}{\partial t}+\frac{\partial \phi}{\partial x}=0
$$

in which the flux $\phi$ is determined as $\phi=v u$, with $v(x, t)$ the transporting velocity. In the present work, 4 classes of nonlinear discretizations for the convective term $\frac{\partial \phi}{\partial x}$ are investigated, i.e. upwind schemes, Total Variation Diminishing (TVD) schemes, Weighted Essentially Non-Oscillatory (WENO) schemes and nonlinear Dynamic Finite Difference (DFD) schemes. For each class, one or more representative examples are selected in order to investigate the nonlinearity in detail. In the current investigation, we select the $1^{s t}, 2^{\text {nd }}$ - and $3^{\text {rd }}$-order upwind scheme, the TVD scheme with the Chakravarthy limiter, the $5^{\text {th }}$-order WENO scheme and the $2^{\text {nd }}$-order nonlinear DFD scheme, for further analysis. These nonlinear finite difference schemes are described below.

1. In general, the discretization of the convective term in a node $i$ of the computational grid with uniform grid spacing $\Delta x$, can be expressed as the differential balance of fluxes at the intermediate node positions $i \pm \frac{1}{2}$, i.e.

$$
\left.\frac{\delta \phi}{\delta x}\right|_{i}=\frac{\phi_{i+\frac{1}{2}}-\phi_{i-\frac{1}{2}}}{\Delta x}
$$

Using the transport velocity $v$ at the intermediate positions $i \pm \frac{1}{2}$, i.e.

$$
v_{i+\frac{1}{2}}=\frac{v_{i}+v_{i+1}}{2} ; \quad v_{i-\frac{1}{2}}=\frac{v_{i}+v_{i-1}}{2}
$$

the $1^{\text {st }}, 2^{\text {nd }}$ and $3^{r d}$-order upwind discretizations for the convective term in (1) are defined respectively as

$$
\begin{aligned}
\left.\frac{\delta \phi}{\delta x}\right|_{i} & =\frac{\left[v_{i+\frac{1}{2}}^{+} u_{i}+v_{i+\frac{1}{2}}^{-} u_{i+1}\right]-\left[v_{i-\frac{1}{2}}^{+} u_{i-1}+v_{i-\frac{1}{2}}^{-} u_{i}\right]}{\Delta} \\
\left.\frac{\delta \phi}{\delta x}\right|_{i} & =\frac{\left[v_{i+\frac{1}{2}}^{+}\left(3 u_{i}-u_{i-1}\right)+v_{i+\frac{1}{2}}^{-}\left(3 u_{i+1}-u_{i+2}\right)\right]-\left[v_{i-\frac{1}{2}}^{+}\left(3 u_{i-1}-u_{i-2}\right)+v_{i-\frac{1}{2}}^{-}\left(3 u_{i}-u_{i+1}\right)\right]}{2 \Delta} \\
\left.\frac{\delta \phi}{\delta x}\right|_{i} & =\frac{\left[v_{i+\frac{1}{2}}^{+}\left(2 u_{i+1}+5 u_{i}-u_{i-1}\right)+v_{i+\frac{1}{2}}^{-}\left(2 u_{i}+5 u_{i+1}-u_{i+2}\right)\right]}{6 \Delta} \\
& -\frac{\left[v_{i-\frac{1}{2}}^{+}\left(2 u_{i}+5 u_{i-1}-u_{i-2}\right)+v_{i-\frac{1}{2}}^{-}\left(2 u_{i-1}+5 u_{i}-u_{i+1}\right)\right]}{6 \Delta},
\end{aligned}
$$

in which $v^{+}=\max (v, 0)$ and $v^{-}=\min (v, 0)$ denote the positive and negative contributions of the transport velocity. It is well known that these upwind discretizations can be interpreted as a combination of a central 
finite difference discretization with a dissipative correction that ensures stability. For example, the first order upwind scheme can be reformulated as

$$
\begin{aligned}
\left.\frac{\delta \phi}{\delta x}\right|_{i} & =\left[\frac{v_{i+\frac{1}{2}} u_{i+1}+\left(v_{i+\frac{1}{2}}-v_{i-\frac{1}{2}}\right) u_{i}-v_{i-\frac{1}{2}} u_{i-1}}{2 \Delta}\right] \\
& -\left[\frac{v_{i+\frac{1}{2}}^{+} u_{i+1}-\left(v_{i+\frac{1}{2}}^{+}+v_{i-\frac{1}{2}}^{+}\right) u_{i}+v_{i-\frac{1}{2}}^{+} u_{i-1}}{2 \Delta}\right]+\left[\frac{v_{i+\frac{1}{2}}^{-} u_{i+1}-\left(v_{i+\frac{1}{2}}^{-}+v_{i-\frac{1}{2}}^{-}\right) u_{i}+v_{i-\frac{1}{2}}^{-} u_{i-1}}{2 \Delta}\right] .
\end{aligned}
$$

Obviously, the nonlinearity in upwind schemes is only activated if the transporting velocity $v$ has positive and negative values in the domain. Hence, for linear convection equations with constant transport velocity $v$, all upwind schemes reduce to standard linear backward $(v>0)$ or forward $(v<0)$ finite difference schemes.

2. Using the same definitions for $v_{i+\frac{1}{2}}^{ \pm}, v_{i-\frac{1}{2}}^{ \pm}$, the TVD discretization of the convective term is defined as

$$
\begin{aligned}
\left.\frac{\delta \phi}{\delta x}\right|_{i} & =\frac{\left[v_{i+\frac{1}{2}}^{+}\left(u_{i}+\frac{\psi\left(r_{i}\right)}{2}\left(u_{i+1}-u_{i}\right)\right)+v_{i+\frac{1}{2}}^{-}\left(u_{i+1}-\frac{\psi\left(\frac{1}{r_{i+1}}\right)}{2}\left(u_{i+1}-u_{i}\right)\right)\right]}{\Delta} \\
& -\frac{\left[v_{i-\frac{1}{2}}^{+}\left(u_{i-1}+\frac{\psi\left(r_{i-1}\right)}{2}\left(u_{i}-u_{i-1}\right)\right)+v_{i-\frac{1}{2}}^{-}\left(u_{i}-\frac{\psi\left(\frac{1}{r_{i}}\right)}{2}\left(u_{i}-u_{i-1}\right)\right)\right]}{\Delta},
\end{aligned}
$$

in which the ratio of consecutive differences in a node $i$ is

$$
r_{i}=\frac{u_{i}-u_{i-1}}{u_{i+1}-u_{i}}
$$

The slope limiter function

$$
\psi(r)=\max (\min (r, 2), 0),
$$

proposed by Chakravarthy et al. [10], is used in this work. If $\psi(r)=0$ (sharp gradient, opposite slopes or zero gradient), the scheme reduces to a $1^{s t}$-order upwind scheme, whereas for $\psi(r)=r$, the scheme is equivalent with a $2^{\text {nd }}$-order upwind scheme. In the particular case of $r=1$ (smooth solution, equal slopes), the scheme turns to a $2^{\text {nd }}$-order central scheme, whereas for $\psi(r)=2$, the scheme switches to a $1^{\text {st }}$-order downwind scheme. Since this limiter function has a large interval in $r$, i.e. $0 \leq r \leq 2$, the scheme reduces to a $2^{\text {nd }}$ -order upwind discretization most of the time. Remark that the Chakravarthy limiter resembles the minmod limiter, which is defined as $\psi(r)=\max (\min (r, 1), 0)$. Similarly to the upwind schemes, the TVD scheme can be reinterpreted as a central scheme in combination with a dissipative correction operator. Due to the limiter function, the dissipative correction is weaker than for a $2^{\text {nd }}$-order upwind scheme. Note that even for a constant transporting velocity $v$, this schemes remains nonlinear.

3. The $5^{\text {th }}$-order Weighted Essentially Non-Oscillatory (WENO) scheme $[4,11]$ for the convective term, is constructed by first splitting the flux $\phi$ in expression (2) into a positive and negative contribution, $\phi^{+}(u)$ and $\phi^{-}(u)$, such that

$$
\left.\frac{\delta \phi}{\delta x}\right|_{i}=\frac{\left(\phi_{i+\frac{1}{2}}^{+}+\phi_{i+\frac{1}{2}}^{-}\right)-\left(\phi_{i-\frac{1}{2}}^{+}+\phi_{i-\frac{1}{2}}^{-}\right)}{\Delta x} .
$$

The flux splitting can be accomplished in different ways. However, often, the Lax-Friederichs flux splitting is used [4], i.e.

$$
\begin{aligned}
\phi^{+}(u) & =\frac{1}{2}(\phi(u)+\theta u)=\frac{1}{2}(v u+\theta u) \\
\phi^{-}(u) & =\frac{1}{2}(\phi(u)-\theta u)=\frac{1}{2}(v u-\theta u),
\end{aligned}
$$


with $\theta=\max \left(\left|\frac{\partial \phi}{\partial u}\right|\right)=\max (|v|)$, over the relevant range. The positive and negative numerical fluxes are obtained as a weighting between 3 basic flux definitions, giving

$$
\begin{aligned}
& \phi_{i+\frac{1}{2}}^{+}=w_{0}^{+}\left[\frac{2 \phi_{i-2}^{+}-7 \phi_{i-1}^{+}+11 \phi_{i}^{+}}{6}\right]+w_{1}^{+}\left[\frac{-\phi_{i-1}^{+}+5 \phi_{i}^{+}+2 \phi_{i+1}^{+}}{6}\right]+w_{2}^{+}\left[\frac{2 \phi_{i}^{+}+5 \phi_{i+1}^{+}-1 \phi_{i+2}^{+}}{6}\right] \\
& \phi_{i+\frac{1}{2}}^{-}=w_{0}^{-}\left[\frac{11 \phi_{i+1}^{-}-7 \phi_{i+2}^{-}+2 \phi_{i+3}^{-}}{6}\right]+w_{1}^{-}\left[\frac{2 \phi_{i}^{-}+5 \phi_{i+1}^{-}-\phi_{i+2}^{-}}{6}\right]+w_{2}^{-}\left[\frac{-\phi_{i-1}^{-}+5 \phi_{i}^{-}+2 \phi_{i+1}^{-}}{6}\right] \\
& \phi_{i-\frac{1}{2}}^{+}=\widetilde{w}_{0}^{+}\left[\frac{2 \phi_{i-3}^{+}-7 \phi_{i-2}^{+}+11 \phi_{i-1}^{+}}{6}\right]+\widetilde{w}_{1}^{+}\left[\frac{-\phi_{i-2}^{+}+5 \phi_{i-1}^{+}+2 \phi_{i}^{+}}{6}\right]+\widetilde{w}_{2}^{+}\left[\frac{2 \phi_{i-1}^{+}+5 \phi_{i}^{+}-1 \phi_{i+1}^{+}}{6}\right] \\
& \phi_{i-\frac{1}{2}}^{-}=\widetilde{w}_{0}^{-}\left[\frac{11 \phi_{i}^{-}-7 \phi_{i+1}^{-}+2 \phi_{i+2}^{-}}{6}\right]+\widetilde{w}_{1}^{-}\left[\frac{2 \phi_{i-1}^{-}+5 \phi_{i}^{-}-\phi_{i+1}^{-}}{6}\right]+\widetilde{w}_{2}^{-}\left[\frac{-\phi_{i-2}^{-}+5 \phi_{i-1}^{-}+2 \phi_{i}^{-}}{6}\right] .
\end{aligned}
$$

In order to achieve a convex combination of Essentially Non-Oscillatory (ENO) stencils, the normalized weighting coefficients $w_{k}^{ \pm}$are determined by

$$
\begin{aligned}
w_{k}^{ \pm} & =\frac{\alpha_{k}^{ \pm}}{\alpha_{0}^{ \pm}+\alpha_{1}^{ \pm}+\alpha_{2}^{ \pm}}, \quad k=0,1,2 \\
\alpha_{k}^{ \pm} & =\frac{c_{k}}{\epsilon+\beta_{k}^{ \pm}}, \quad k=0,1,2,
\end{aligned}
$$

in which the $\beta_{k}^{ \pm}$represent local indicators of the smoothness of the flux, and $\epsilon=10^{-6}$ is a small positive number, necessary to avoid singularities in the method. For the $5^{\text {th }}$-order WENO scheme, $c_{0}=\frac{1}{10}, c_{1}=\frac{6}{10}, c_{2}=\frac{3}{10}$. The smoothness indicators are determined by the relations

$$
\begin{aligned}
& \beta_{0}^{+}=\frac{13}{12}\left[\phi_{i-2}^{+}-2 \phi_{i-1}^{+}+\phi_{i}^{+}\right]^{2}+\frac{1}{4}\left[\phi_{i-2}^{+}-4 \phi_{i-1}^{+}+3 \phi_{i}^{+}\right]^{2} \\
& \beta_{1}^{+}=\frac{13}{12}\left[\phi_{i-1}^{+}-2 \phi_{i}^{+}+\phi_{i+1}^{+}\right]^{2}+\frac{1}{4}\left[\phi_{i-1}^{+}-\phi_{i+1}^{+}\right]^{2} \\
& \beta_{2}^{+}=\frac{13}{12}\left[\phi_{i}^{+}-2 \phi_{i+1}^{+}+\phi_{i+2}^{+}\right]^{2}+\frac{1}{4}\left[3 \phi_{i}^{+}-4 \phi_{i+1}^{+}+\phi_{i+2}^{+}\right]^{2} \\
& \beta_{0}^{-}=\frac{13}{12}\left[\phi_{i+1}^{-}-2 \phi_{i+2}^{-}+\phi_{i+3}^{-}\right]^{2}+\frac{1}{4}\left[3 \phi_{i+1}^{-}-4 \phi_{i+2}^{-}+\phi_{i+3}^{-}\right]^{2} \\
& \beta_{1}^{-}=\frac{13}{12}\left[\phi_{i}^{-}-2 \phi_{i+1}^{-}+\phi_{i+2}^{-}\right]^{2}+\frac{1}{4}\left[\phi_{i}^{-}-\phi_{i+2}^{-}\right]^{2} \\
& \beta_{2}^{-}=\frac{13}{12}\left[\phi_{i-1}^{-}-2 \phi_{i}^{-}+\phi_{i+1}^{-}\right]^{2}+\frac{1}{4}\left[\phi_{i-1}^{-}-4 \phi_{i}^{-}+3 \phi_{i+1}^{-}\right]^{2} .
\end{aligned}
$$

Analogous relations are obtained for $\widetilde{w}_{k}^{ \pm}, \widetilde{\alpha}_{k}^{ \pm}$and $\widetilde{\beta}_{k}^{ \pm}$. Note that in contrast to the upwind schemes, WENO schemes remain nonlinear even for constant transport velocity $v$.

4. The last nonlinear finite difference scheme that is investigated in this work, is the central nonlinear DFD scheme of Fauconnier et al. [6]. This scheme is defined by

$$
\left.\frac{\delta \phi}{\delta x}\right|_{i}=\frac{\left[\phi_{i+1}+c_{i+1}\left(\phi_{i+2}-2 \phi_{i+1}+\phi_{i}\right)\right]-\left[\phi_{i-1}+c_{i-1}\left(\phi_{i}-2 \phi_{i-1}+\phi_{i-2}\right)\right]}{2 \Delta}+\mathscr{O}\left(\Delta^{2}\right),
$$

with $\phi=v u$. The dynamic coefficient $c$ is determined via the transported scalar $u(x)$ as [6]

$$
c_{i}=-\frac{1}{6} \frac{1}{1+f \max \left(\min \left(\frac{u_{i+2}-4 u_{i+1}+6 u_{i}-4 u_{i-1}+u_{i-2}}{u_{i+1}-2 u_{i}+u_{i-1}}, 0\right),-3\right)} .
$$


The parameter $f$ is obtained by calibrating the scheme such that it obtains maximum accuracy for a field with a prescribed inertial range spectrum defined in expression (28). This leads to values around $f=0.21$. It was shown in [6], that for $f=0.2$, the nonlinear DFD scheme has the same modified wavenumber as the standard $6^{\text {th }}$-order tridiagonal compact scheme, for a single-wave field. Therefore, we will adopt $f=0.2$ in this work. For more information about the mathematical derivation and justification of the nonlinear DFD scheme, we refer to Fauconnier et al. [6]. This scheme does not depend on a transporting velocity, and thus remains nonlinear in each point for a constant transport velocity $v$. We emphasize that, in contrast to the upwind schemes, the TVD scheme and the WENO scheme, the DFD scheme (26) consists of a central discretization operator in combination with a dispersive correction operator. As mentioned in the introduction, the goal of the DFD scheme is to increase the accuracy of the discretization by adding a dispersive correction, instead of preserving the stability by adding a dissipative operator to the basic discretization. Hence, the spectral behaviour of the upwind schemes, the TVD scheme and the WENO scheme on the one hand, and the nonlinear DFD scheme on the other hand, will be substantially different and must not be compared. Here, the only objective is to examine the influence of the nonlinearity on e.g. the spectral characteristics, not comparison of the spectral characteristics between the various schemes defined above.

\section{Nonlinear Spectral Analysis}

\subsection{Methodology}

For the selected nonlinear schemes, we first investigate the impact of the nonlinearity on the modified wavenumber and the energy spectrum, by means of an artificial or synthetic field for $u(x)$, containing a predefined spectrum of Fourier modes. The one-dimensional synthetic field $u(x), 0 \leq x<2 \pi$ is constructed such that its energy spectrum $E_{u}(\kappa)$ follows a predefined inertial range scaling

$$
E_{u}(\kappa) \propto\left\{\begin{array}{ll}
\kappa^{\alpha} & 0<\kappa \leq \kappa_{c} \\
0 & \kappa_{c}<\kappa \leq \kappa_{\max }
\end{array},\right.
$$

whereas the phase $\varphi_{u}(\kappa), 0 \leq \kappa \leq \kappa_{c}$, of each mode is chosen randomly in the interval $[-\pi,+\pi]$ according to a uniform distribution $\mathscr{U}[-\pi,+\pi]$, i.e.

$$
\varphi_{u}(\kappa)=\left\{\begin{array}{ll}
\mathscr{U}[-\pi,+\pi] & 0<\kappa \leq \kappa_{c} \\
0 & \kappa_{c}<\kappa \leq \kappa_{\max }
\end{array} .\right.
$$

In the previous expressions, $\kappa_{\max }=\frac{\pi}{\Delta}$ denotes the maximum wavenumber of an equidistant computational grid with grid spacing $\Delta$. Defining the Fourier transform of $u(x)$ as

$$
\widehat{u}(\kappa)=\mathscr{F}\{u(x)\}=a(\kappa)+i b(\kappa),
$$

the synthetic field is then determined by satisfying the conditions

$$
\begin{aligned}
& E_{u}(\kappa)=a(\kappa)^{2}+b(\kappa)^{2}=U_{0} \kappa^{\alpha}, \quad 0<\kappa \leq \kappa_{c} \\
& \varphi_{u}(\kappa)=\arctan \left(\frac{b(\kappa)}{a(\kappa)}\right), \quad 0<\kappa \leq \kappa_{c},
\end{aligned}
$$

in which $U_{0}$ is a proportionality factor that can be chosen freely. At $\kappa=0$ we impose $E_{u}(0)=0$, i.e. the mean of $u(x)$ is zero. Extracting $a(\kappa)$ and $b(\kappa)$, leads to the expression of the synthetic field in Fourier space

$$
\widehat{u}(\kappa)=\left\{\begin{array}{ll} 
\pm \sqrt{U_{0}} \frac{\left(1+i \tan \left(\varphi_{u}(\kappa)\right)\right)}{\sqrt{1+\tan ^{2}\left(\varphi_{u}(\kappa)\right)}} \kappa^{\alpha / 2} & 0<\kappa \leq \kappa_{c} \\
0+i 0 & \kappa_{c}<\kappa \leq \kappa_{\max }
\end{array} .\right.
$$

Taking the inverse Fourier transform, one readily obtains the synthetic field in physical space $u(x)$. Once the field $u(x)$ is obtained, the modified wavenumber $\kappa^{\prime}$ of any linear or nonlinear discretization $\frac{\delta u}{\delta x}$ of the first derivative, is determined as

$$
\kappa^{\prime}(\kappa)=\frac{1}{\widehat{\imath u}(\kappa)} \mathscr{F}\left\{\frac{\delta u}{\delta x}\right\}, \quad \forall \kappa \leq \kappa_{c} .
$$


For linear schemes, analytical expressions can easily be derived for the modified wavenumber. For nonlinear schemes, however, such analytical expressions are prohibitively complex, involving the evaluation of convolution integrals. Hence, expression (34) is considered as a good and workable alternative. The phase function $\varphi_{u}(\kappa)$ in expression (33) must be considered as a particular $k^{\text {th }}$ random realization $\varphi_{k}(\kappa)$ of a uniform distribution of the phase in the interval $[-\pi, \pi]$. Hence, the modified wavenumber $\kappa^{\prime}=\kappa^{\prime}\left(\kappa, \varphi_{k}\right)$ depends on that particular realization $\varphi_{k}(\kappa)$ of the phase function $\varphi_{u}(\kappa)$. Therefore, it is useful to provide statistical information about $\kappa^{\prime}$ as well, such as the mean modified wavenumber and the variance. This is achieved by determining the modified wavenumber for $N_{r}$ realizations of the synthetic field $u(x)$, and calculating respectively the mean modified wavenumber, and the standard deviation as

$$
\begin{aligned}
\mu_{\kappa^{\prime}}(\kappa) & =\frac{1}{N_{r}} \sum_{k=0}^{N_{r}} \kappa^{\prime}\left(\kappa, \varphi_{k}\right) \\
\sigma_{\kappa^{\prime}}(\kappa) & =\left\{\frac{1}{N_{r}-1} \sum_{k=0}^{N_{r}}\left[\kappa^{\prime}\left(\kappa, \varphi_{k}\right)-\mu_{\kappa^{\prime}}(\kappa)\right]^{2}\right\}^{1 / 2} .
\end{aligned}
$$

In the following, we consider the slope of the turbulent energy spectrum, i.e. $\alpha=-5 / 3$. The cutoff wavenumber is chosen systematically as the maximum wavenumber of the computational grid, i.e. $\kappa_{c}=\kappa_{\max }=\frac{\pi}{\Delta}$. In some particular situations, we also investigate $\kappa_{c}=\frac{2}{3} \kappa_{\max }$, which matches the dealiasing limit of Orszag [12]. The statistical information is extracted, using $N_{r}=10^{5}$ realizations of the synthetic field. This is enough to ensure that the mean and the variation are representative. In other words, taking more realizations does not alter significantly $\mu_{\kappa^{\prime}}(\kappa)$ nor $\sigma_{\kappa^{\prime}}(\kappa)$. Aside from the analysis of the modified wavenumber, it is also interesting to investigate the impact of the nonlinearity on the energy spectrum of the synthetic field. This allows to study the energetic impact of the nonlinear mechanisms and to visualize the spurious scales that are generated by the nonlinear schemes at the tail of the energy spectrum $[8,1]$. In analogy with the analytical relation

$$
\widehat{\partial u}=i \widehat{\alpha u},
$$

we can define a reconstructed synthetic field $u_{r}(x)$ as

$$
\frac{\widehat{\delta u}}{\delta x}=i \kappa \widehat{u}_{r}
$$

Then the energy spectrum of the reconstructed field is obtained as

$$
\begin{aligned}
E_{u_{r}}\left(\kappa, \varphi_{k}\right) & =\frac{1}{\kappa^{2}} E_{\frac{\delta u}{\delta x}}\left(\kappa, \varphi_{k}\right), \quad \forall \kappa \\
& = \begin{cases}\frac{\kappa^{2}}{\kappa^{2}} E_{u}\left(\kappa, \varphi_{k}\right) & 0<\kappa \leq \kappa_{c} \\
\text { spurious modes } & \kappa_{c}<\kappa \leq \kappa_{\max }\end{cases}
\end{aligned}
$$

Obviously, the energy spectrum of $u_{r}$ is directly proportional to the energy spectrum of the derivative. Remark that the reconstructed energy spectrum depends on the particular realization $\varphi_{k}$ of the phase function $\varphi_{u}(\kappa)$. Hence we determine again statistical information, i.e. a mean energy spectrum and the variance on the energy spectrum, using

$$
\begin{aligned}
\mu_{E_{u_{r}}}(\kappa) & =\frac{1}{N_{r}} \sum_{k=0}^{N_{r}} E_{u_{r}}\left(\kappa, \varphi_{k}\right) \\
\sigma_{E_{u_{r}}}(\kappa) & =\left\{\frac{1}{N_{r}-1} \sum_{k=0}^{N_{r}}\left[E_{u_{r}}\left(\kappa, \varphi_{k}\right)-\mu_{E_{u_{r}}}(\kappa)\right]^{2}\right\}^{1 / 2} .
\end{aligned}
$$

In the following, we refer to the described methodology as a Nonlinear Spectral Analysis (NSA). 

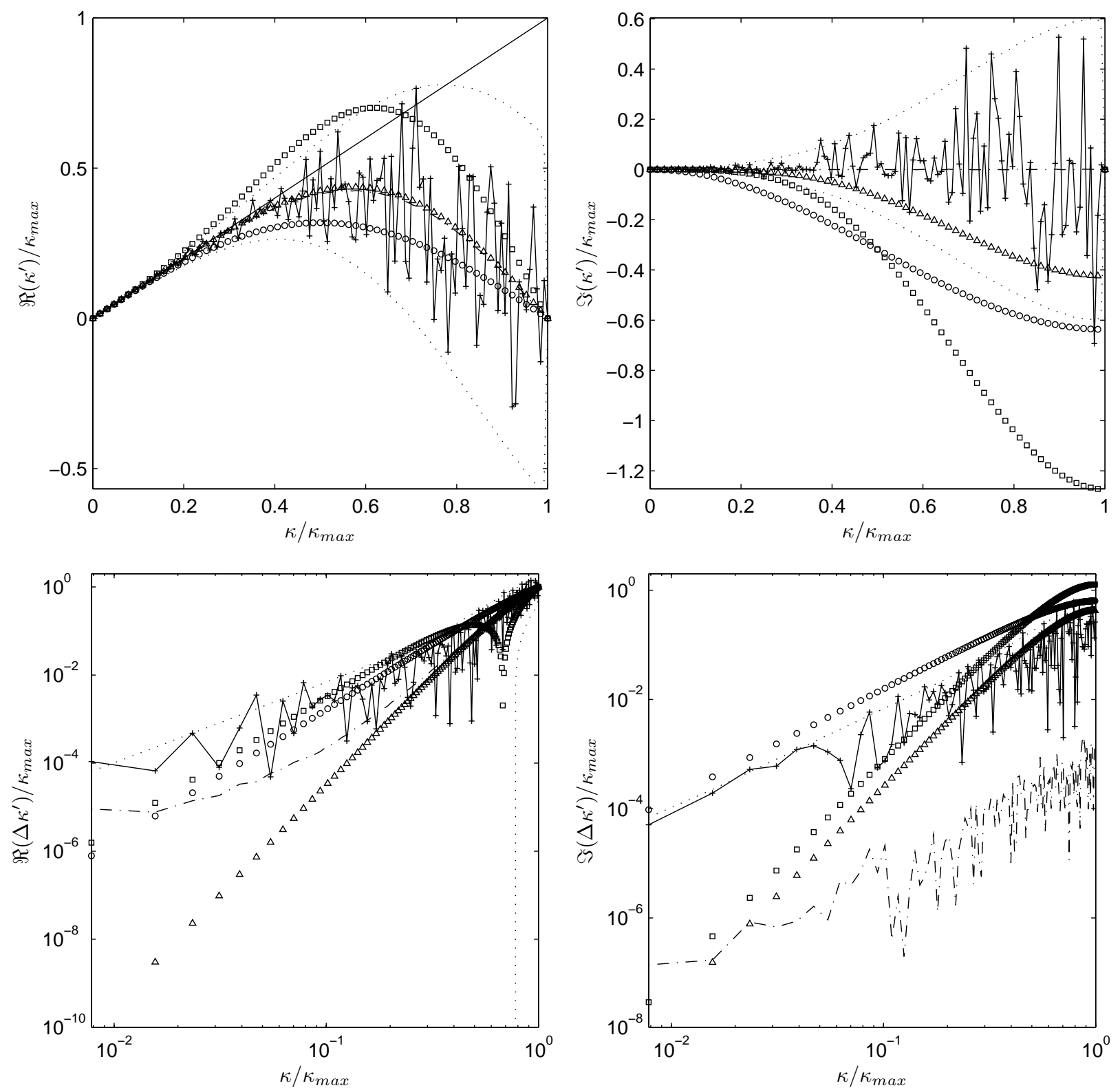

Figure 1: Modified wavenumber for the $3^{\text {rd }}$-order upwind scheme: Dispersion characteristic (upperleft); dissipation characteristic (upperright). Error on the dispersion characteristic (lowerleft); error on the dissipation characteristic (lowerright). $1^{\text {st }}$-order backward (o); $2^{\text {nd }}$-order backward $(\square) ; 3^{r d}$-order backward $(\Delta) ; \kappa^{\prime}\left(\kappa, \varphi_{k}\right)(+) ; \mu_{\kappa^{\prime}}(\kappa)(-\cdot-\cdot) ; \mu_{\kappa^{\prime}}(\kappa) \pm 2 \sigma_{\kappa^{\prime}}(\kappa)(\cdots)$. 


\subsection{Analysis}

Before the Nonlinear Spectral Analysis can be applied to the different nonlinear schemes, defined in section 2, the discrete derivative $\frac{\delta u}{\delta x}$ of the synthetic field $u(x)$, used e.g. in expression (34), must be defined properly. Therefore, we choose the transporting velocity $v$ in the flux definition $\phi$ of equation (1) to be unity in the entire domain. Indeed, the nonlinear TVD scheme, the $5^{\text {th }}$-order WENO scheme and the DFD scheme for $\frac{\delta \phi}{\delta x}$, given by expressions (8), (11) and (26), reduce to the equivalent nonlinear discretizations for $\frac{\delta u}{\delta x}$. However, since the nonlinearity in upwind schemes (4), (5) and (6) is only activated if the transporting velocity $v$ has positive and negative values in the domain, the choice $v=1$, reduces all upwind schemes to standard linear backward finite difference schemes. As a consequence, the Nonlinear Spectral Analysis, proposed in section 3.1, is redundant for upwind schemes when assuming a constant $v$, because the modified wavenumber can then be obtained analytically. For the Nonlinear Spectral Analysis of the upwind schemes in this work, we intentionally assume that $v^{+}=|\max (\operatorname{sgn}(u), 0)|$ and $v^{-}=|\min (\operatorname{sgn}(u), 0)|$, in order to have an idea about the impact of the nonlinearity on the modified wavenumber. In the current analysis, we only focus on the $3^{\text {rd }}$-order upwind scheme.

The NSA results for the $3^{\text {rd }}$-order upwind scheme, the TVD-scheme, the $5^{\text {th }}$-order WENO-scheme and the nonlinear DFD scheme are shown in Figures 1, 2, 4 and 5. The results show the instantaneous modified wavenumber $\kappa^{\prime}\left(\kappa, \varphi_{k}\right)$ for the arbitrary $k^{t h}$ synthetic field realization with $\kappa_{c}=\kappa_{\max }$, the mean modified wavenumber $\mu_{\kappa^{\prime}}(\kappa)$ for $N_{r}=10^{5}$ synthetic field realizations, and the spreading of the results bounded by the lines $\mu_{\kappa^{\prime}}(\kappa) \pm 2 \sigma_{\kappa^{\prime}}(\kappa)$. Further, also the error on the modified wavenumber, and the spreading of the error around the mean is shown on a logarithmic scale, in order to focus on the asymptotic behaviour of the schemes in the low wavenumber range. For the $3^{\text {rd }}$-order upwind scheme, the TVD-scheme and the $5^{\text {th }}$-order WENO scheme, the spectral characteristics are compared with those of the $1^{\text {st }}-, 2^{\text {nd }}$ - and $3^{\text {rd }}$-order backward finite difference schemes, whereas for the DFD scheme, we compare the characteristics with those of the $4^{\text {th }}$ - and $6^{\text {th }}$-order central schemes and the $6^{\text {th }}$-order tridiagonal compact scheme. Figures 2, 4 also show the quasi-linear Approximate Dispersion Relation (ADR) of Pirozzoli [1], respectively for the TVD-scheme with the minmod and Chakravarthy limiters, and for the $5^{\text {th }}$-order WENO-scheme.

First, we consider the $3^{\text {rd }}$-order upwind scheme in figure 1. It is seen that both the real and imaginary part of the modified wavenumber $\kappa^{\prime}\left(\kappa, \varphi_{k}\right)$ display a very erratic behaviour, which increases with increasing wavenumber. Looking at the dispersion property, the mean modified wavenumber $\mu_{\kappa^{\prime}}(\kappa)$ collapses, as expected, with the modified wavenumber of the $3^{r d}$-order backward scheme in almost the entire wavenumber range. However, the error on the real part reveals that $3^{r d}$-order scaling is lost if $\kappa \rightarrow 0$. In fact, both $\kappa^{\prime}\left(\kappa, \varphi_{k}\right)$ and $\mu_{\kappa^{\prime}}(\kappa)$ tend to the scaling of the $1^{s t}$ and $2^{\text {nd }}$-order backward schemes. For the dissipation property, the mean modified wavenumber $\mu_{\kappa^{\prime}}(\kappa)$ is nearly zero in the entire wavenumber range, although the discretization stencil is never unbiased. This somewhat misleading result stems from the choice to define synthetic fields, with zero mean component, and use it for this particular upwind case as the transporting velocity. In other words, since the field $u(x)$ has a zero mean, the $3^{r d}$-order forward discretization stencil is applied as much as the backward discretization stencil, resulting in an average zero-dissipation. Hence, if the mean component was slightly positive, then, the mean modified wavenumber $\mu_{\kappa^{\prime}}(\kappa)$ would shift more towards that of the $3^{\text {rd }}$-order backward scheme. The error on the imaginary part reveals that in the asymptotic range $\kappa \rightarrow 0, \kappa^{\prime}\left(\kappa, \varphi_{k}\right)$ and $\mu_{\kappa^{\prime}}(\kappa)$ obtain the scaling of the $1^{s t}$ - and $2^{\text {nd }}$-order backward schemes. Based on Figure 1, one is tempted to assume that the dispersion and the dissipation characteristics are quasi randomly distributed around the mean modified wavenumber $\mu_{\kappa^{\prime}}(\kappa)$. Based on the standard deviation, however, it is clear that for approximately $95 \%$ of all realizations, $\kappa^{\prime}\left(\kappa, \varphi_{k}\right)$ is confined to the area between the curves $\mu_{\kappa^{\prime}}(\kappa) \pm 2 \sigma_{\kappa^{\prime}}(\kappa)$. The spreading is quite large, indicating that occurrences of a negative dispersion relation is possible in the range $\kappa>0.6 \kappa_{\max }$. The spreading on the dissipation relation, shows that the dissipation can be higher than that of a $3^{\text {rd }}$-order forward or backward scheme, in the entire wavenumber range. Moreover, in the low wavenumber range, the dissipation is close to that of a $1^{s t}$ order forward or backward scheme. The conclusions for other upwind schemes, which are not shown, are very similar.

Next, we investigate the TVD-scheme in figure 2. Again, we notice that both the real and imaginary parts of the modified wavenumber $\kappa^{\prime}\left(\kappa, \varphi_{k}\right)$ display erratic behaviour, similarly to the upwind schemes. Looking at the dispersion property, the mean modified wavenumber $\mu_{\kappa^{\prime}}(\kappa)$ (almost) collapses with the modified wavenumber of the $3^{\text {rd }}$-order backward scheme in the medium and high wavenumber range. However, the error on the real part reveals that the 

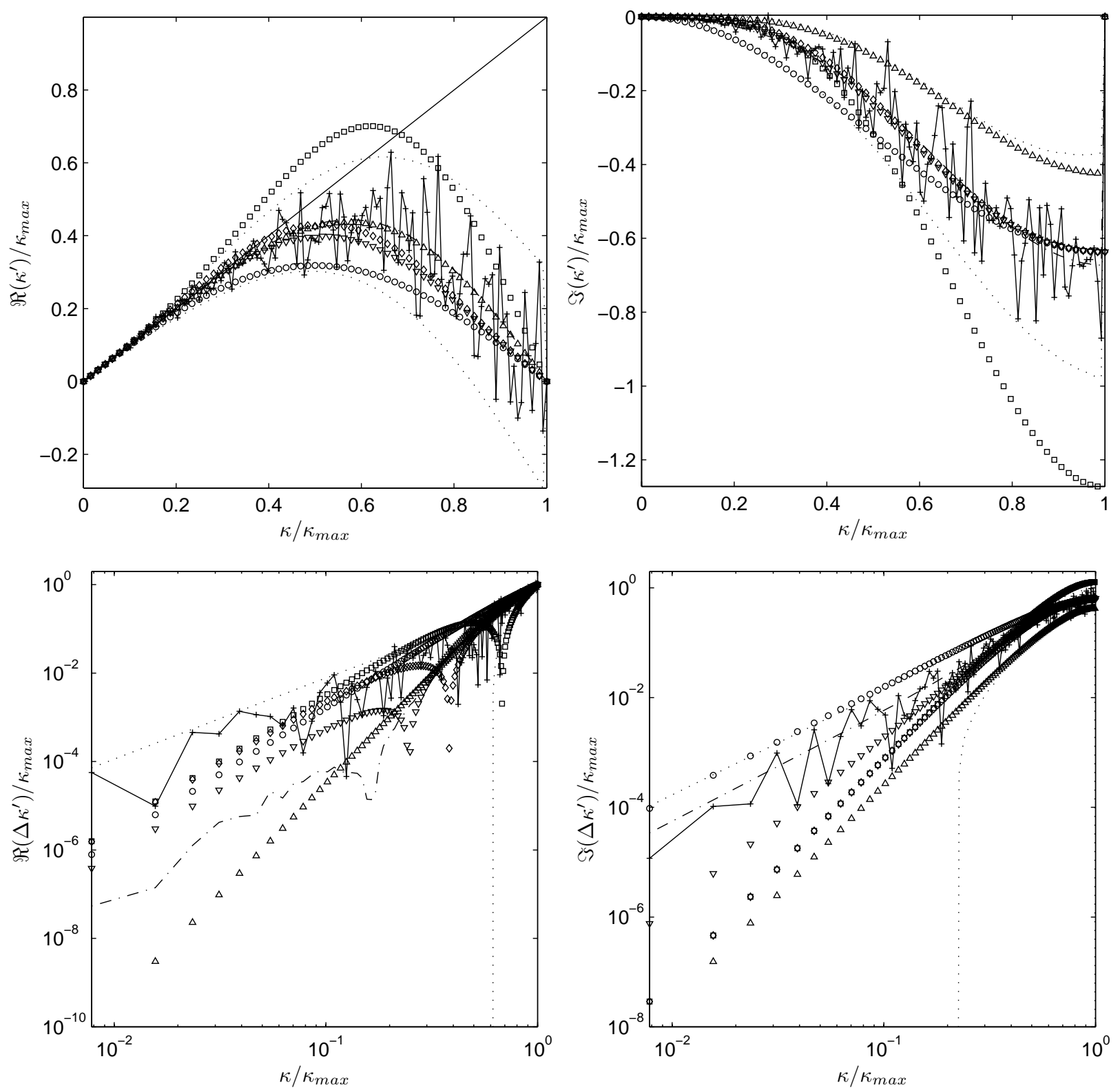

Figure 2: Modified wavenumber for the TVD scheme: Dispersion characteristic (upperleft); dissipation characteristic (upperright). Error on the dispersion characteristic (lowerleft); error on the dissipation characteristic (lowerright). $1^{\text {st }}$-order backward (o); $2^{\text {nd }}$-order backward $(\square) ; 3^{r d}$-order backward $(\triangle)$; ADR of TVD + minmod limiter [1] ( $\left.\nabla\right)$; ADR of TVD + Chakravarthy limiter $(\diamond) ; \kappa^{\prime}\left(\kappa, \varphi_{k}\right)(+) ; \mu_{\kappa^{\prime}}(\kappa)(-\cdot-\cdot)$; $\mu_{\kappa^{\prime}}(\kappa) \pm 2 \sigma_{\kappa^{\prime}}(\kappa)(\cdots)$. 

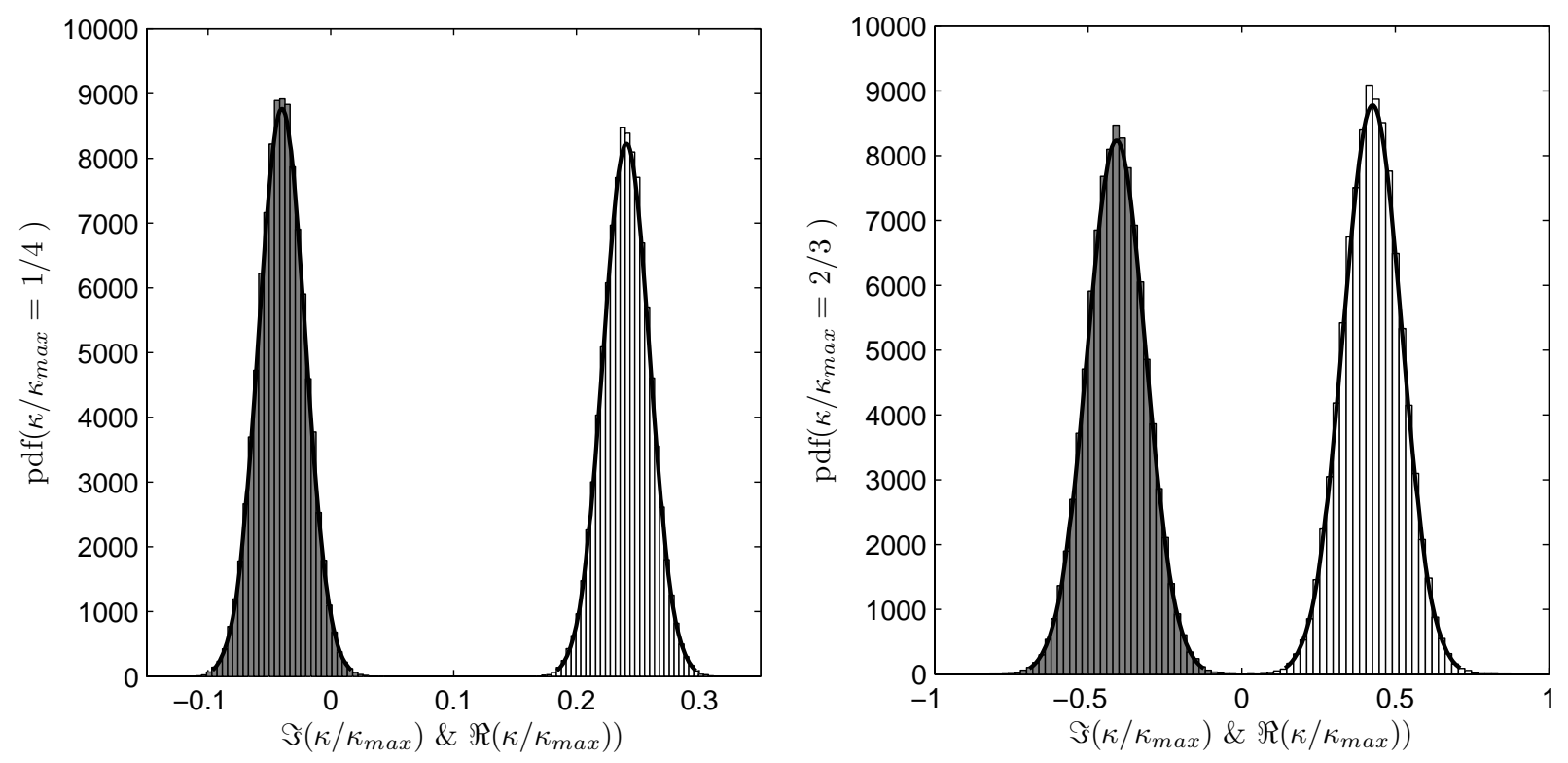

Figure 3: Distribution of modes for the TVD scheme: distribution at wavenumber $\kappa / \kappa_{\max }=1 / 4$ (left) and $\kappa / \kappa_{\max }=2 / 3$ (right). Histograms represents the distribution of the dispersion characteristic (light grey), and the distribution of the dissipation characteristic (dark grey).

accuracy is not maintained in the asymptotic range $\kappa \rightarrow 0$. In fact, both $\kappa^{\prime}\left(\kappa, \varphi_{k}\right)$ and $\mu_{\kappa^{\prime}}(\kappa)$ tend to the scaling of the $1^{\text {st }}$ - and $2^{\text {nd }}$-order backward schemes. Remark that the quasi-linear ADR results of Pirozzoli [1] for the dispersion relation of the TVD-scheme with limiter (10), are clearly different from the mean dispersion relation obtained with the NSA. For the dissipation property, the mean modified wavenumber $\mu_{\kappa^{\prime}}(\kappa)$ follows a specific path in between the modified wavenumbers of the $1^{\text {st }}$ - and $3^{\text {rd }}$-order backward schemes in the medium and high wavenumber range. Again, the error on the imaginary part reveals that in the asymptotic range $\kappa \rightarrow 0, \kappa^{\prime}\left(\kappa, \varphi_{k}\right)$ and $\mu_{\kappa^{\prime}}(\kappa)$ tend to a $1^{s t}$ -order scaling. Note that for the dissipation, the quasi-linear ADR results of Pirozzoli [1] are surprisingly close to the results obtained with the NSA. Further, the spreading around the mean modified wavenumber is significant, indicating that occurrences of a negative dispersion relation are possible for $\kappa>0.6 \kappa_{\max }$. Moreover, the spreading shows that in the high wavenumber range, the numerical dissipation could become smaller than that of a $3^{\text {rd }}$-order backward scheme, but could also become significantly larger than that of a $1^{\text {st }}$-order backward scheme. In order to have a good idea of the real distribution of $\kappa^{\prime}\left(\kappa, \varphi_{k}\right)$ around its mean, the Probability Density Function is shown in Figure 3 for two wavenumbers, i.e. $\kappa / \kappa_{\max }=1 / 4$ and $\kappa / \kappa_{\max }=2 / 3$. The bell-shaped curves clearly indicate a normal or Gaussian distribution of the modified wavenumber around the mean. We conclude that the mean dispersive and dissipative properties of the TVD scheme behave as expected in the medium and high-wavenumber range, despite reduction of the order of accuracy for $\kappa \rightarrow 0$. The latter, caused by the nonlinearity of the scheme, is in contrast with the formal $2^{\text {nd }}$-order accuracy, aimed at in the construction of the TVD-scheme.

For the $5^{\text {th }}$-order WENO scheme, erratic behaviour is again observed for both the real and imaginary parts of the modified wavenumber $\kappa^{\prime}\left(\kappa, \varphi_{k}\right)$. Looking at the dispersion property, the mean modified wavenumber $\mu_{\kappa^{\prime}}(\kappa)$ does not reach the accuracy of the $3^{r d}$-order backward scheme in the entire wavenumber range. Moreover, the error on the real part reveals that in the asymptotic range $\kappa \rightarrow 0$, the scaling of $\kappa^{\prime}\left(\kappa, \varphi_{k}\right)$ and $\mu_{\kappa^{\prime}}(\kappa)$ tend to only the scaling of the $1^{s t}$ - and $2^{\text {nd }}$-order backward schemes. These results are in contrast to the quasi-linear ADR results of Pirozzoli [1] for the $5^{\text {th }}$-order WENO scheme, which show a $5^{\text {th }}$-order scaling for $\kappa \rightarrow 0$. Note that the scheme is constructed to have a $5^{\text {th }}$-order accuracy. In the high wavenumber range, the NSA predicts a much better behaviour of the dispersion relation than the quasi-linear ADR, although it does not reach the quality of the $3^{\text {rd }}$-order scheme. For the dissipation property, the mean modified wavenumber $\mu_{\kappa^{\prime}}(\kappa)$ lies between the modified wavenumbers of the $1^{s t}-$ and $3^{\text {rd }}$-order backward schemes in the medium and high wavenumber range. The error on the imaginary part shows that 

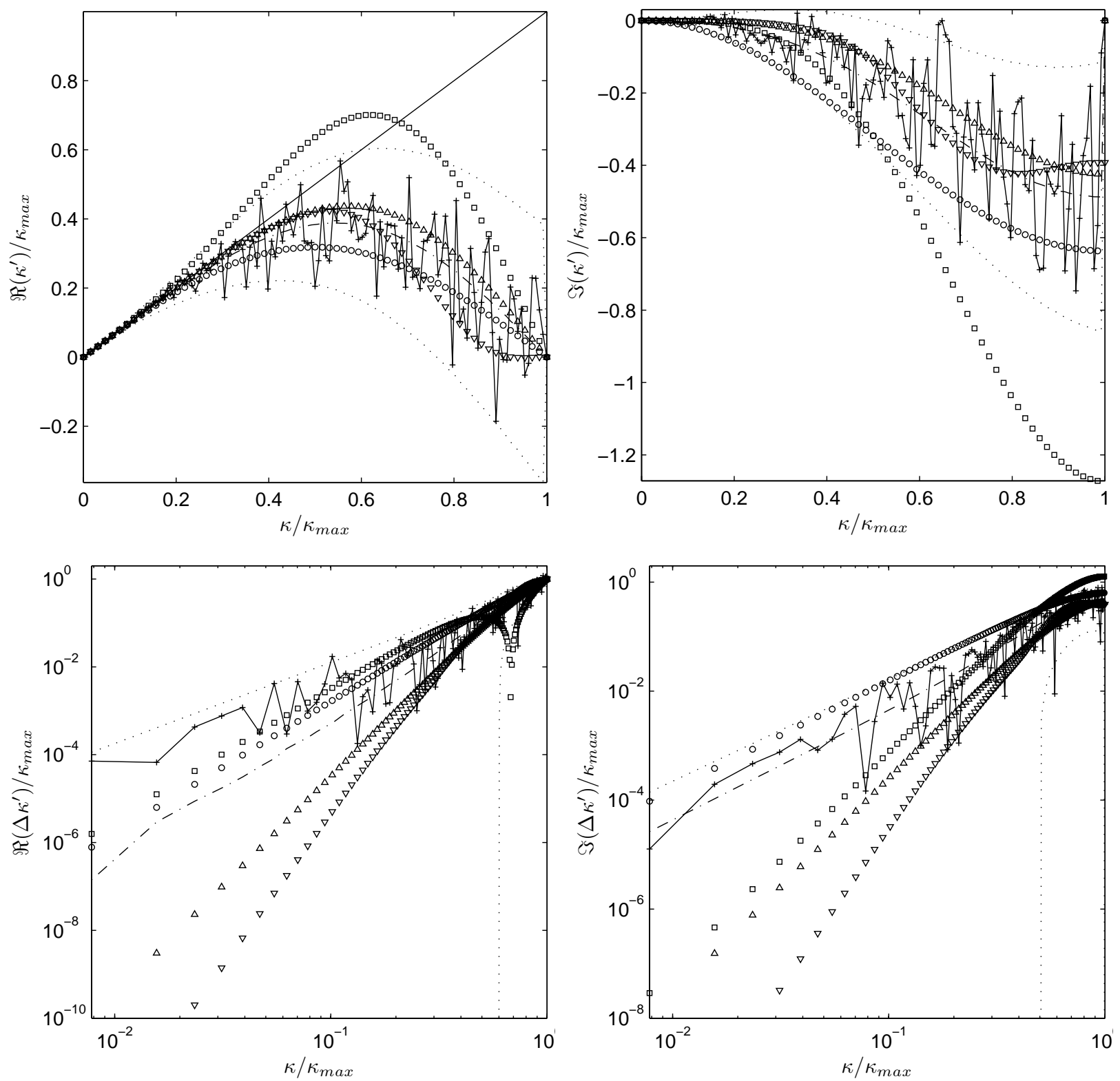

Figure 4: Modified wavenumber for the $5^{\text {th }}$-order WENO scheme: Dispersion characteristic (upperleft); dissipation characteristic (upperright). Error on the dispersion characteristic (lowerleft); error on the dissipation characteristic (lowerright). $1^{s t}$-order backward (०); $2^{\text {nd }}$-order backward ( $\left.\square\right) ; 3^{r d}$-order backward $(\triangle)$; ADR of $5^{\text {th }}$-order WENO scheme $[1](\nabla) ; \kappa^{\prime}\left(\kappa, \varphi_{k}\right)(+) ; \mu_{\kappa^{\prime}}(\kappa)(-\cdot-\cdot) ; \mu_{\kappa^{\prime}}(\kappa) \pm 2 \sigma_{\kappa^{\prime}}(\kappa)(\cdots)$. 
for $\kappa \rightarrow 0, \kappa^{\prime}\left(\kappa, \varphi_{k}\right)$ and $\mu_{\kappa^{\prime}}(\kappa)$ tend to a $1^{s t}$-order scaling, which is in contrast with the quasi-linear ADR results of Pirozzoli [1]. Indeed, the ADR results, show a different behaviour of the dissipation characteristics in comparison with the NSA results, in the entire wavenumber range. This emphasizes the importance of contributions of nonlinear interactions to the spectral properties. The spreading around the mean modified wavenumber indicates that negative dispersion can occur for $\kappa>0.6 \kappa_{\max }$. The dissipation can become lower than that of a $3^{r d}$-order backward scheme, but can also be significantly larger than that of a $1^{\text {st }}$-order backward scheme. We conclude that the dispersive and dissipative properties of the $5^{\text {th }}$-order WENO scheme are strongly determined by the nonlinear mechanism, resulting in different spectral characteristics in the entire wavenumber range, than intended by construction, or expected from linear analysis. The Probability Density Function shows a normal distribution of the modified wavenumber around the mean, similarly to that of the TVD scheme. Therefore it is not shown.

Finally, we consider the nonlinear DFD-scheme in figure 5. Once more, one observes the erratic behaviour of the modified wavenumber $\kappa^{\prime}\left(\kappa, \varphi_{k}\right)$, that increases with increasing wavenumber. In comparison with the upwind schemes, the TVD scheme and the WENO scheme, the amplitude of these disturbances is much smaller. Looking at the dispersion property, the mean modified wavenumber $\mu_{\kappa^{\prime}}(\kappa)$ does not collapse with the characteristic of the $6^{\text {th }}$ -order tridiagonal compact scheme, which is in contrast to the conclusion from the analytical single-wave analysis in the work of Fauconnier et al. [6]. In the high wavenumber range, the mean dispersion relation $\mu_{\kappa^{\prime}}(\kappa)$ displays a pretty good behaviour, as it is better than that of the $6^{\text {th }}$-order central scheme, although, it does not reach the accuracy of the $6^{\text {th }}$-order tridiagonal compact scheme. However, the error on the real part reveals once more that the accuracy is not maintained in the asymptotic range $\kappa \rightarrow 0$. Whereas $\mu_{\kappa^{\prime}}(\kappa)$ tends to $2^{\text {nd }}$-order scaling, $\kappa^{\prime}\left(\kappa, \varphi_{k}\right)$ seem to indicate only $1^{s t}$-order scaling. For the dissipation property, the mean modified wavenumber $\mu_{\kappa^{\prime}}(\kappa)$ is very close to zero, which is not surprising since the nonlinear DFD has a central discretization stencil, regardless the value of the preceding coefficient $c$. Hence in the mean, no dissipation should occur. However, the small symmetric spreading predicts an equal probability for a small amount of dissipation or anti-dissipation of the scheme for any Fourier mode. In the asymptotic range $\kappa \rightarrow 0, \kappa^{\prime}\left(\kappa, \varphi_{k}\right)$ and $\mu_{\kappa^{\prime}}(\kappa)$ display $1^{s t}$-order scaling. The Probability Density Function learned again that the modified wavenumber is normally distributed, and is therefore not shown. For the nonlinear DFD scheme we conclude that the dispersion properties are of significant lower quality for small wavenumbers than one would theoretically expect from the construction of this scheme. Again this is due to the nonlinearity.

As discussed in section 3.1, the energy spectra of the reconstructed fields give us some more information about the impact of the nonlinearity on the magnitude of the different Fourier modes, and the generation of spurious scales above the cutoff wavenumber. Figure 6 shows these energy spectra for respectively the TVD-scheme, the $5^{\text {th }}$-order WENO scheme and the DFD scheme, using cutoff wavenumbers $\kappa_{c}=\kappa_{\max }$ and $\kappa_{c}=\kappa_{\max } / 2$. All plots show that the nonlinearity is responsible for spurious disturbances in the entire spectrum of Fourier modes. As a consequence, some modes are energized, whereas others are damped. Moreover, the scattering of these disturbances is relatively large around the cutoff wavenumber $\kappa_{c}$, as indicated by the curves $\mu_{E_{u r}}(\kappa) \pm 2 \sigma_{E_{u r}}(\kappa)$. Hence, the smallest regular modes are affected the most by this nonlinear behaviour. In the case $\kappa_{c} / \kappa_{\max }=1 / 2$, one clearly notices the creation of spurious modes above the cutoff wavenumber $\kappa_{c}$. This supports the conclusion of both Ladeinde et al. [8], who stated that ENO and WENO schemes generate numerical turbulence, and Fauconnier et al. [6] who found similar results for the nonlinear DFD scheme. For the TVD scheme and the WENO scheme, these spurious scales are approximately two magnitude smaller than the smallest scales at the cutoff wavenumber, whereas for the DFD scheme, the spurious modes are approximately four magnitudes smaller than the smallest scales at the cutoff wavenumber. Hence, the relative importance of these spurious modes in comparison with the regular modes, seem to depend strongly on the type of discretization. Note that in case of a linear or nonlinear convection equation, these spurious modes interact with all other modes. This may have a severe impact on the evolution of the solution. Moreover, the interactions of the spurious scales in the wavenumber region $\kappa_{c} \leq \kappa_{\max }$ inevitably lead to aliasing errors, affecting even more the evolution of the solution. These issues will be investigated later in this work.

\section{Conservation properties of nonlinear schemes}

In the discussion above, it was shown that the nonlinear mechanisms have a strong impact on the modified wavenumber and the energy spectrum of the reconstructed synthetic field. Not only does it seem to affect all ex- 

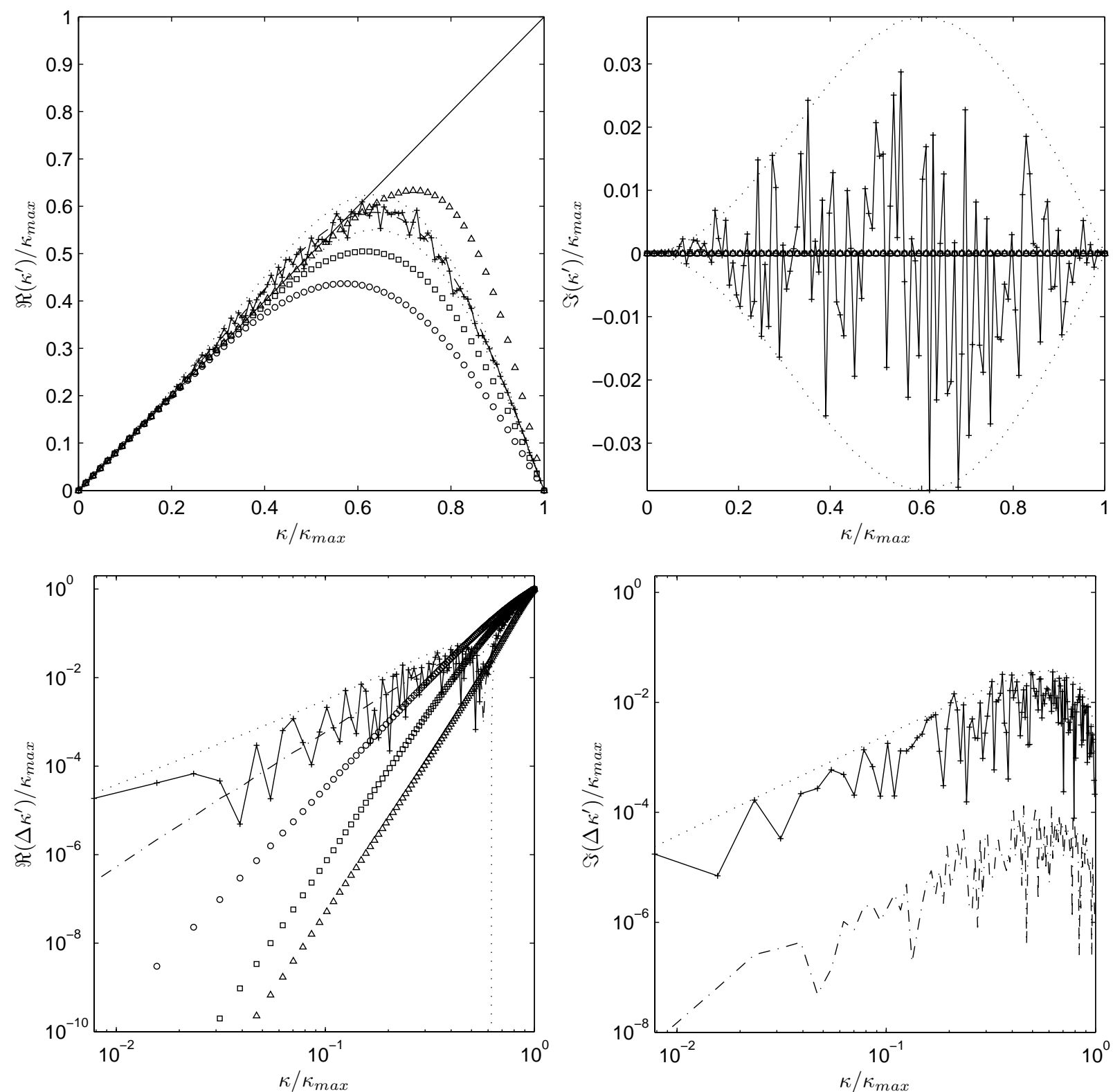

Figure 5: Modified wavenumber for the DFD scheme: Dispersion characteristic (upperleft); dissipation characteristic (upperright). Error on the dispersion characteristic (lowerleft); error on the dissipation characteristic (lowerright). $4^{\text {th }}$-order central (o); $6^{\text {th }}$-order central ( $\square$ ); $6^{\text {th }}$-order tridiagonal compact $(\Delta) ; \kappa^{\prime}\left(\kappa, \varphi_{k}\right)(+) ; \mu_{\kappa^{\prime}}(\kappa)(-\cdot-\cdot) ; \mu_{\kappa^{\prime}}(\kappa) \pm 2 \sigma_{\kappa^{\prime}}(\kappa)(\cdots)$. 

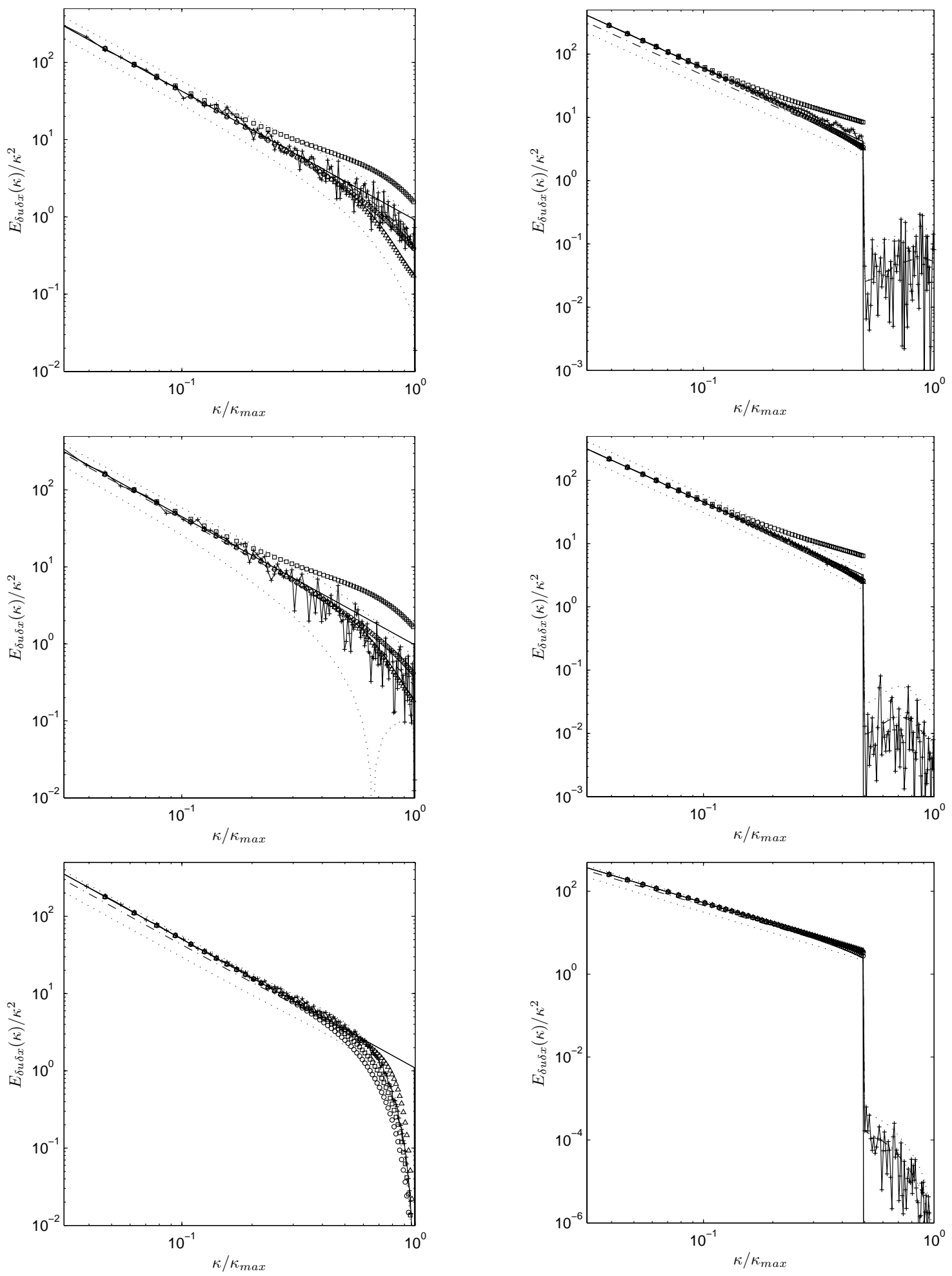

Figure 6: Energy spectrum of $u_{r}: \kappa_{c} / \kappa_{\max }=1$ (left column); $\kappa_{c} / \kappa_{\max }=1 / 2$ (right column). TVD scheme $\left(1^{\text {st }}\right.$ row); $5^{\text {th }}$-order WENO scheme $\left(2^{\text {nd }}\right.$ row); DFD scheme ( $3^{r d}$ row). Symbols for the TVD and WENO schemes: $\kappa^{-5 / 3}(-)$ ); $1^{\text {st }}$-order upwind (o); $2^{\text {nd }}$-order upwind ( $\left.\square\right) ; 3^{r d}$ -order upwind $(\triangle)$. Symbols for the DFD-scheme: $\kappa^{-5 / 3}(-) ; 4^{\text {th }}$-order central (o); $6^{\text {th }}$-order central $(\square) ; 6^{\text {th }}$-order tridiagonal compact $(\triangle)$. Scheme of interest: $E_{u_{r}}\left(\kappa, \varphi_{k}\right)(+) ; \mu_{E_{u_{r}}}(\kappa)(-\cdot-\cdot) ; \mu_{E_{u_{r}}}(\kappa) \pm 2 \sigma_{E_{u_{r}}}(\kappa)(\cdots)$. 
isting scales, i.e. large scales as well as small scales, it also creates spurious modes that were not present in the original synthetic field. This immediately raises some questions about the conservation of momentum, and more importantly, the net conservation of energy during a simulation. Therefore, it is interesting to verify the effect of the nonlinear mechanism on the conservation properties, in order to know its impact on the solution in a real simulation. In particular the net energy contribution of the nonlinearity to the energy balance is of concern.

In the following, we investigate the conservation properties of nonlinear schemes. We restrict ourselves in this work to the analysis of the elementary, though relevant, one-dimensional linear convection equation. Analysis of the conser-

vation properties in the one-dimensional and linear case is an essential prerequisite before analyzing conservation for more-dimensional and/or nonlinear cases. If conservation is violated in a linear, one-dimensional setup, conservation will certainly fail in nonlinear and higher-dimensional setups. Note that because the linear convection equation is assumed, the current analysis is not directly representative for upwind schemes. In this work we follow the approach of Wissink [13], as it is convenient for our purposes.

\subsection{One-dimensional linear convection equation}

Consider the following linear convection equation for the periodically transported field $u(x, t), x \in[0,2 \pi], t \geq 0$

$$
\frac{\partial u}{\partial t}+v \frac{\partial u}{\partial x}=0
$$

in which $v$ represents the constant convective transport velocity. The global conservation of momentum $u(x, t)$ is expressed as

$$
\frac{d}{d t} \int_{0}^{2 \pi} u(x, t) d x=\int_{0}^{2 \pi} \frac{\partial u}{\partial t} d x=0
$$

whereas the global conservation of the kinetic energy $u(x, t)^{2} / 2$ is given by

$$
\frac{d}{d t} \int_{0}^{2 \pi} \frac{u(x, t)^{2}}{2} d x=\int_{0}^{2 \pi} u \frac{\partial u}{\partial t} d x=0 .
$$

Using expression (43), both expressions are reformulated as

$$
\int_{0}^{2 \pi} v \frac{\partial u}{\partial x} d x=0, \quad \forall t \geq 0
$$

and

$$
\int_{0}^{2 \pi} v u \frac{\partial u}{\partial x} d x=0, \quad \forall t \geq 0
$$

Assuming a non-uniform grid with $N$ nodes $x_{i}, i \in \mathbb{N}$, the continuum expressions are written discretely as

$$
\left.\sum_{i} v \frac{\delta u}{\delta x}\right|_{i} \Delta x_{i}=0, \quad \forall t \geq 0,
$$

and

$$
\left.\sum_{i} v u_{i} \frac{\delta u}{\delta x}\right|_{i} \Delta x_{i}=0, \quad \forall t \geq 0 .
$$


where $\Delta x_{i}$ denotes the integration weight which gives an approximation of the grid spacing at node $i, \forall i$.

In order to derive the conservation properties of nonlinear schemes, we define the discrete derivative $\frac{\delta u}{\delta x}$ in a node $i$ as

$$
\left.\frac{\delta u}{\delta x}\right|_{i}=\sum_{j=-r}^{r} \beta_{i, j} u_{i+j}
$$

in which the coefficients $\beta_{i, j}$ determine the stencil in a particular node $i$. Obviously, this formulation accounts for the nodal variation of the discretization stencils, which is the main issue of interest in this work. The only condition stencil $\beta_{i, j}$ is assumed to satisfy, is that it gives an approximation for the first derivative. This implies that the trivial condition that the derivative of a constant field should be exact, i.e. $\sum_{j=-r}^{r} \beta_{i, j}=0, \forall i$. It is emphasized that the summation over $j=-r \cdots r$ does not necessarily imply that the discretization is central, since the integer $r$ merely denotes the maximum of the right and left stencil widths. We further define the stencil width as $w=2 r+1$. Using definition (50), the discrete conservation expressions (48) and (49) are written as

$$
\sum_{i} \sum_{j=-r}^{r} \beta_{i, j} u_{i+j} \Delta x_{i}=0, \quad \forall t \geq 0,
$$

and

$$
\sum_{i} \sum_{j=-r}^{r} \beta_{i, j} u_{i+j} u_{i} \Delta x_{i}=0, \quad \forall t \geq 0 .
$$

Working out (51) by collecting the factors for each node $i$, that is, changing the index notations in expression (51) by replacing index $i+j$ with $i$ (and as a consequence $i$ with $i-j$ ), and replacing afterwards the index $-j$ with $j$, one obtains the expression for the global conservation of momentum

$$
\sum_{i}\left(\sum_{j=-r}^{r} \beta_{i+j,-j} \Delta x_{i+j}\right) u_{i}=0, \quad \forall t \geq 0 .
$$

Repeating the latter procedure for expression (52), yields

$$
\sum_{i} \sum_{j=-r}^{r} \beta_{i+j,-j} u_{i} u_{i+j} \Delta x_{i+j}=0, \quad \forall t \geq 0 .
$$

Obviously, the conditions (52) and (54) for the conservation of kinetic energy must be satisfied at the same time, since they share the mutual term $u_{i} u_{i+j}$. Hence, their sum must also be equal to zero, leading to following expression for the global conservation of kinetic energy

$$
\sum_{i} \sum_{j=-r}^{r}\left(\beta_{i, j} \Delta x_{i}+\beta_{i+j,-j} \Delta x_{i+j}\right) u_{i} u_{i+j}=0, \quad \forall t \geq 0 .
$$

First we focus on expression (53) for the global momentum conservation. In order to satisfy expression (53) for any arbitrary realization of $u\left(x_{i}\right)$, the vector with elements $\sum_{j=-r}^{r} \beta_{i+j,-j} \Delta x_{i+j}$ must satisfy any linear combination of the Null Space of $u\left(x_{i}\right)$, i.e. Null $\left(\left[u_{1}, \ldots, u_{n}\right]\right)$, where the Null Space is a set of $n-1$ basis vectors with size $n \times 1$, each satisfying (53). Thus, for $n-1$ arbitrary weighting coefficients, the corresponding linear combination of Null $\left(\left[u_{1}, \ldots, u_{n}\right]\right)$, results in $n$ conditions for the coefficients $\sum_{j=-r}^{r} \beta_{i+j,-j} \Delta x_{i+j}, \forall i$. Hence, this implies that in order to satisfy global conservation of momentum, the momentum deficit in $n-1$ computational cells must be compensated in the remaining single cell. In other words, any linear combination of the Null Space, gives a sufficient condition for global momentum conservation. However, local conservation of momentum requires that each cell has a zero loss of momentum. In other words, only the trivial solution, in which $n-1$ weighting coefficients are chosen exactly zero, gives a sufficient and necessary condition for local momentum conservation. Obviously, local conservation also 
guarantees global conservation in a periodic domain. Hence, we conclude that the necessary and sufficient condition for local conservation of momentum is given by

$$
\sum_{j=-r}^{r} \beta_{i+j,-j} \Delta x_{i+j}=0, \quad \forall i .
$$

Applying the same reasoning to expression (55), leads to the conclusion that the necessary and sufficient condition for local conservation of kinetic energy is given by

$$
\begin{aligned}
\beta_{i, 0} \Delta x_{i} & =0, \quad \forall i \\
\beta_{i, j} \Delta x_{i}+\beta_{i+j,-j} \Delta x_{i+j} & =0, \quad \forall i, j .
\end{aligned}
$$

Assume now a coefficient matrix $\gamma$

$$
\gamma_{i, i+j}=\beta_{i, j} \Delta x_{i}, \quad \forall i, \forall-r \leq j \leq r .
$$

Note that in a periodic domain with $n$ nodes, positions $i+j<1$, correspond to $n+i+j$ whereas positions $i+j>n$ correspond to $i+j-n$. Then, condition (56) for the momentum conservation expresses that the sum of each column in this coefficient matrix $\gamma$ must be zero. This is known as the telescoping property. Condition (57) for the energy conservation expresses that the diagonal of the coefficient matrix $\gamma$ must be zero, whereas condition (58) states that matrix $\gamma$ must be skew-symmetric, i.e.

$$
\gamma=-\gamma^{T}
$$

Although the stencil coefficients $\beta_{i, j}$ can vary from node to node, conditions (56), (57) and (58) impose certain restrictions, as shown hereafter.

Considering again the nonlinear schemes defined in section (2), it is understood that all of these schemes can be reinterpreted somehow as a spatial weighting of a series of linear (node-independent) flux definitions. In analogy with this observation, we continue with the very general assumption that a general discretization stencil $\beta_{i, j}$ of the derivative could be interpreted as a spatial weighting of a series of node-independent stencils, defined on an uniform grid. For the sake of simplicity, we restrict ourselves to a spatial weighting of two basic discretizations $\beta_{j}^{(1)}$ and $\beta_{j}^{(2)}$, giving

$$
\beta_{i, j}=p_{i, i+j} \beta_{j}^{(1)}+q_{i, i+j} \beta_{j}^{(2)},
$$

in which $p_{i, i+j}$ and $q_{i, i+j}$ represent the weighting functions that depend on the node index $i$ as well as on the discretization index $j$. At this point, the only condition to which the standard, Taylor-based finite difference discretizations $\beta_{j}^{(1)}$ and $\beta_{j}^{(2)}$ are subjected, is that their combination (61) must result into a valid approximation for the first derivative. Substituting (61) into expression (56) for the momentum conservation, yields

$$
\sum_{j=-r}^{r}\left(p_{i+j, i} \beta_{-j}^{(1)}+q_{i+j, i} \beta_{-j}^{(2)}\right) \Delta x_{i+j}=0, \quad \forall i .
$$

Analogously, substitution of (61) into expressions (57) and (58) for the conservation of kinetic energy yields

$$
\begin{aligned}
p_{i, i} \beta_{0}^{(1)} \Delta x_{i}+q_{i, i} \beta_{0}^{(2)} \Delta x_{i} & =0, \quad \forall i \\
p_{i, i+j} \beta_{j}^{(1)} \Delta x_{i}+p_{i+j, i} \beta_{-j}^{(1)} \Delta x_{i+j}+q_{i, i+j} \beta_{j}^{(2)} \Delta x_{i}+q_{i+j, i} \beta_{-j}^{(2)} \Delta x_{i+j} & =0, \quad \forall i, j .
\end{aligned}
$$

\subsubsection{Momentum conservation}

First we focus on the condition (62) for the momentum conservation. Assume that the standard linear discretization stencils $\beta_{j}^{(1)}$ and $\beta_{j}^{(2)}$ both satisfy condition (56) for the conservation of momentum on an equidistant grid, such that

$$
\sum_{j=-r}^{r} \beta_{-j}^{(1)}=0, \quad \sum_{j=-r}^{r} \beta_{-j}^{(2)}=0, \quad \forall i .
$$


Condition (62) is then satisfied, if the factors $p_{i+j, i} \Delta x_{i+j}$ and $q_{i+j, i} \Delta x_{i+j}$ are independent of index $j, \forall i$. In other words, for a particular index $i$, the weightings $p_{i+j, i} \Delta x_{i+j}$ and $q_{i+j, i} \Delta x_{i+j}$ must be constant in $j$. However, both weightings do not have to be constant in $i$. Assuming that

$$
\begin{aligned}
p_{i+j, i} \Delta x_{i+j} & =p_{i, i}^{\prime} \\
q_{i+j, i} \Delta x_{i+j} & =q_{i, i}^{\prime},
\end{aligned}
$$

one obtains the following relation for the weighting functions

$$
\begin{aligned}
p_{i+j, i} & =\frac{p_{i, i}^{\prime}}{\Delta x_{i+j}} \\
q_{i+j, i} & =\frac{q_{i, i}^{\prime}}{\Delta x_{i+j}}, .
\end{aligned}
$$

Transformation of these expressions to index $(i, i+j)$, i.e. replacing index $i$ with $i-j$ and afterwards $-j$ with $j$, results finally into the conditions

$$
\begin{aligned}
& p_{i, i+j}=\frac{p_{i+j, i+j}^{\prime}}{\Delta x_{i}} \\
& q_{i, i+j}=\frac{q_{i+j, i+j}^{\prime}}{\Delta x_{i}} .
\end{aligned}
$$

Obviously, the weighting functions $p_{i, i+j}$ and $q_{i, i+j}$ in definition (61) must include the contribution $\Delta x_{i}^{-1}$ which compensates for the grid non-uniformity, since the basic discretization stencils $\beta_{j}^{(1)}$ and $\beta_{j}^{(2)}$ do not account for this. Apart from that, the remaining weighting of the two basic discretizations must be written as

$$
\beta_{i, j}=\frac{1}{\Delta x_{i}}\left[p_{i+j, i+j}^{\prime} \beta_{j}^{(1)}+q_{i+j, i+j}^{\prime} \beta_{j}^{(2)}\right] .
$$

in order to conserve momentum. This means that the coefficient matrix is given by

$$
\gamma_{i, i+j}=p_{i+j, i+j}^{\prime} \beta_{j}^{(1)}+q_{i+j, i+j}^{\prime} \beta_{j}^{(2)}, \quad \forall i, \forall-r \leq j \leq r .
$$

Hence, all weightings $p_{i+j, i+j}^{\prime}$ and $q_{i+j, i+j}^{\prime}$ in a specific column $i+j$, must have a constant value, equal to that of the diagonal element at position $(i+j, i+j)$.

In conclusion, any weighting of two or more basic finite difference approximations results in a loss of momentum conservation, unless it is expressed as (72). Obviously, the nonlinear finite difference schemes (4), (5), (6), (8), (11) and (26), defined in section (2), are expressed in the divergence formulation, and thus satisfy the conditions for conservation of momentum.

\subsubsection{Energy conservation}

For the analysis of the conditions (63) and (64) for energy conservation, we assume that the standard finite difference discretization stencils $\beta_{j}^{(1)}$ and $\beta_{j}^{(2)}$ both satisfy conditions (57) and (58) for the conservation of kinetic energy on an equidistant grid, such that

$$
\begin{aligned}
& 0=\beta_{0}^{(1)}=\beta_{0}^{(2)}, \\
& 0=\beta_{j}^{(1)}+\beta_{-j}^{(1)}=\beta_{j}^{(2)}+\beta_{-j}^{(2)} .
\end{aligned}
$$

Note that this implies that both standard discretizations are central and skew-symmetric. It is obvious that under this assumption, condition (63) is satisfied automatically. However, the only reasonable condition to satisfy (64) $\forall i$, is

$$
\begin{array}{rlr}
p_{i, i+j} \Delta x_{i} & =p_{i+j, i} \Delta x_{i+j} & \forall i, j \\
q_{i, i+j} \Delta x_{i} & =q_{i+j, i} \Delta x_{i+j} & \forall i, j .
\end{array}
$$


Elimination of the grid non-uniformity by assuming

$$
\begin{aligned}
p_{i, i+j} & =\frac{p_{i, i+j}^{\prime}}{\Delta x_{i}} \\
q_{i, i+j} & =\frac{q_{i, i+j}^{\prime}}{\Delta x_{i}},
\end{aligned}
$$

results in the conditions

$$
\begin{aligned}
p_{i, i+j}^{\prime} & =p_{i+j, i}^{\prime} \quad \forall i, j \\
q_{i, i+j}^{\prime} & =q_{i+j, i}^{\prime} \quad \forall i, j .
\end{aligned}
$$

Obviously, these conditions for the kinetic energy conservation, are only satisfied if the weighting functions $p_{i, i+j}^{\prime}$ and $q_{i, i+j}^{\prime}$ are constants, i.e. $p^{\prime}$ and $q^{\prime}$. Hence, the weighting functions $p_{i, i+j}$ and $q_{i, i+j}$ must only account for the grid non-uniformity. In conclusion, varying the discretization stencil from node to node on a uniform or non-uniform computational grid, results always in a loss of kinetic energy conservation, regardless the conservation properties of the standard finite difference stencils in each node, because the skew-symmetry demand for the coefficient matrix $\gamma$ is violated. In other words, nonlinear mechanisms in discretizations will always result in a loss of kinetic energy conservation. As a consequence, none of the nonlinear finite difference schemes discussed in this work conserves kinetic energy locally, nor globally.

\subsubsection{Integration weights on non-uniform grids}

So far, we showed that any weighting of two (by extension more) basic conservative node-independent discretizations, must be of the form

$$
\beta_{i, j}=\frac{1}{\Delta x_{i}}\left[p^{\prime} \beta_{j}^{(1)}+q^{\prime} \beta_{j}^{(2)}\right]
$$

in order to conserve momentum and kinetic energy. In previous sections, the exact form of the integration weight $\Delta x_{i}$, which gives an approximation of the grid spacing at node $i$ was not yet specified. Following Wissink [13], one may obtain an expression for $\Delta x_{i}$ by demanding that the discrete derivative should be exact for any first-order polynomial, i.e. $u_{i}=a x_{i}+b$, such that

$$
\left.\frac{\delta u}{\delta x}\right|_{i}=\sum_{j=-r}^{r} \beta_{i, j}\left(a x_{i+j}+b\right)=a,
$$

and thus

$$
\sum_{j=-r}^{r} \frac{1}{\Delta x_{i}}\left[p^{\prime} \beta_{j}^{(1)}+q^{\prime} \beta_{j}^{(2)}\right] x_{i+j}=1 .
$$

It then follows immediately from expression (84) that

$$
\Delta x_{i}=\sum_{j=-r}^{r}\left[p^{\prime} \beta_{j}^{(1)}+q^{\prime} \beta_{j}^{(2)}\right] x_{i+j} .
$$

\subsubsection{Energy Balance}

In the previous sections, we analytically derived the conservation requirements for any spatially nonlinear weighting of two standard discretization schemes. We proved that it is not possible to conserve the kinetic energy in case of nonlinear schemes, even if the discretization stencils are central. In other words, one cannot prevent the nonlinearity to have a non-zero contribution to the energy balance. In the current section, we investigate whether the nonlinear mechanism leads to a positive or negative net energy contribution, and more importantly, how the average behaviour 
is.

Consider therefore the change of kinetic energy in time, i.e. the dissipation rate

$$
\frac{d k}{d t}=\frac{d}{d t} \int_{0}^{2 \pi} \frac{u(x, t)^{2}}{2} d x=\int_{0}^{2 \pi} u \frac{\partial u}{\partial t} d x, \quad \forall t \geq 0 .
$$

Substitution of equation (43), results in

$$
\frac{d k}{d t}=-\int_{0}^{2 \pi} v u \frac{\partial u}{\partial x} d x, \quad \forall t \geq 0 .
$$

For any energy-conserving discretization, the dissipation must equal zero. For the sake of simplicity, we assume further an equidistant grid with grid spacing $\Delta x$. Moreover, we assume that the field $u(x, t)$ is a band-limited field, such that all Fourier modes, identified by $\widehat{u}(\kappa, t)$, can be represented on the computational grid. In order to investigate the contribution of the nonlinearity to the kinetic energy, we apply the Nonlinear Spectral Analysis on the expression for the evolution equation (87).

Consider convection equation (43). Using a spectral Fourier method for the spatial derivative, equation (43) can be represented exactly in discrete Fourier space as

$$
\frac{d \widehat{u}}{d t}(\kappa, t)=-v i \kappa \widehat{u}(\kappa, t)
$$

whereas the corresponding equation for the complex conjugate in discrete Fourier space is

$$
\frac{d \widehat{u}^{*}}{d t}(\kappa, t)=v i \widehat{u}^{*}(\kappa, t)
$$

Using a finite difference approximation for the spatial derivative, the convection equations for $\widehat{u}$ and $\widehat{u}^{*}$, can be represented in discrete Fourier space as

$$
\begin{aligned}
\frac{d \widehat{u}}{d t}(\kappa, t) & =-v i \kappa \widehat{u}_{r}(\kappa, t) \\
\frac{d \widehat{u}^{*}}{d t}(\kappa, t) & =v i \kappa \widehat{u}_{r}^{*}(\kappa, t),
\end{aligned}
$$

in which the reconstructed field $u_{r}(x)$ for the discrete derivative was defined in subsection 3.1 , and is given by the relation

$$
\widehat{\delta u}=i \kappa \widehat{u}_{r} .
$$

Further, the evolution of the kinetic energy for each Fourier mode is now obtained by

$$
\frac{d k}{d t}(\kappa, t)=\frac{1}{2} \frac{d \widehat{u} \widehat{u}^{*}}{d t}=\frac{1}{2} \widehat{u} \frac{d \widehat{u}^{*}}{d t}+\frac{1}{2} \widehat{u}^{*} \frac{d \widehat{u}}{d t} .
$$

Substitution of equations (90) and (91) in the previous expression yields

$$
\frac{d k}{d t}(\kappa, t)=\frac{v}{2} i \kappa\left[\widehat{u} \widehat{u}_{r}^{*}-\widehat{u}^{*} \widehat{u}_{r}\right]
$$

Note that this expression is equivalent with

$$
\frac{d k}{d t}(\kappa, t)=\frac{v}{2} \widehat{u} \widehat{u}^{*}\left[\kappa^{* *}-\kappa^{\prime}\right],
$$



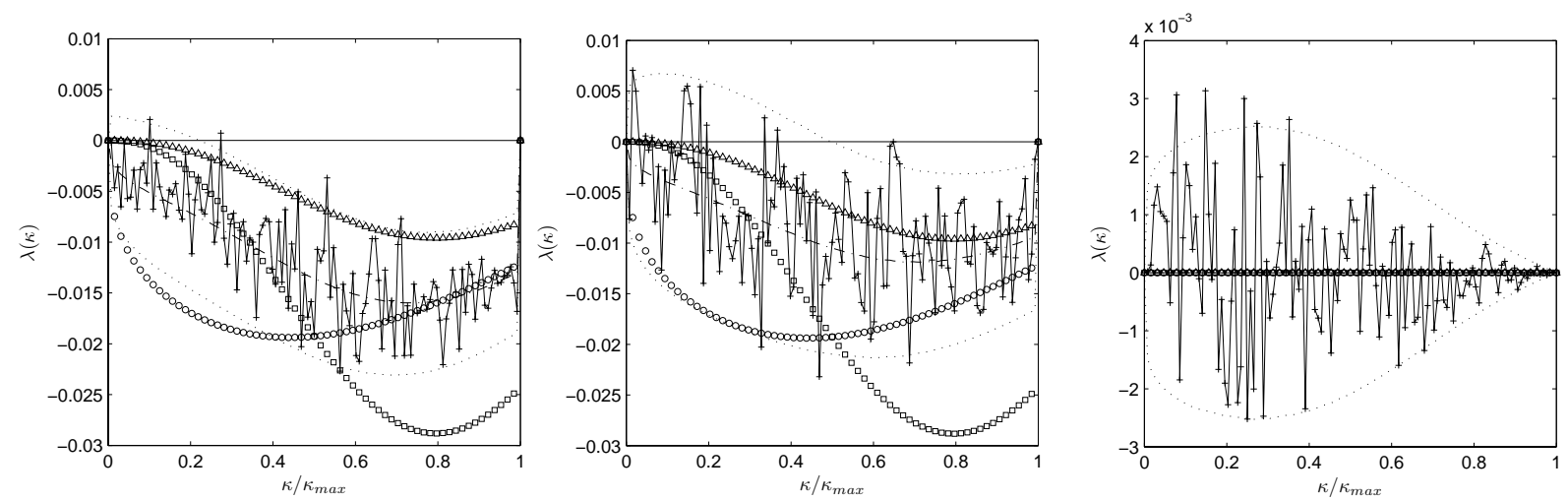

Figure 7: Spectral distribution of the normalized dissipation $\lambda(\kappa)$. TVD scheme (left); $5^{\text {th }}$-order WENO scheme (center); DFD scheme (right). Symbols, see figures 2, 4 and 5 .

in which $\kappa^{\prime *}$ represents the modified wavenumber of the finite difference scheme. However, we prefer the first expression, since for nonlinear schemes, the modified wavenumber becomes singular for $\kappa>\kappa_{c}$, due to expression (34), whereas the reconstructed field $u_{r}(x)$, is well defined in the entire wavenumber range. Defining the initial kinetic energy

$$
k_{0}=\frac{1}{2} \int_{-\kappa_{\max }}^{\kappa_{\max }} \widehat{u} \widehat{u}^{*} d \kappa
$$

the evolution equation for the kinetic energy per Fourier mode is normalized and rearranged, resulting into the expression

$$
\frac{1}{v k_{0}} \frac{d k}{d t}(\kappa, t)=\lambda(\kappa)=\frac{i \kappa\left[\widehat{u} \widehat{u}_{r}^{*}-\widehat{u}^{*} \widehat{u}_{r}\right]}{\int_{-\kappa_{\max }}^{\kappa_{\max }} \widehat{u} \widehat{u}^{*} d \kappa},
$$

whereas the evolution of the total kinetic energy is given as

$$
\frac{1}{v k_{0}} \frac{d k}{d t}(t)=\lambda=\frac{\int_{-\kappa_{\max }}^{\kappa_{\max }} i \kappa\left[\widehat{u} \widehat{u}_{r}^{*}-\widehat{u}^{*} \widehat{u}_{r}\right] d \kappa}{\int_{-\kappa_{\max }}^{\kappa_{\max }} \widehat{u} \widehat{u}^{*} d \kappa} .
$$

It is easy to verify that the right-hand side of both expressions (97) and (98) is always real. Obviously, in case of a spectral discretization with $\widehat{u}_{r}=\widehat{u}$, no kinetic energy is lost. Moreover, expression (95) shows that in case of a central linear discretization, for which the modified wavenumber has no imaginary part, the kinetic energy is conserved. It is necessary to emphasize that this analysis is only valid for a single time-step advancement, and does not take into account the nonlinear interactions that will lead to a deformation the original transported wave during time-advancement.

The function $\lambda(\kappa)$ represents the spectral distribution of a normalized dissipation rate, whereas $\lambda$ represents the total normalized dissipation rate. Both quantities can be used to quantify the loss/gain of kinetic energy. In analogy with the NSA in section 3, a statistical analysis can be made of the normalized dissipation spectrum or the total normalized dissipation for $N_{r}=10^{5}$ realizations of a synthetic field with spectrum scaling $\kappa^{-5 / 3}$ and cutoff wavenumber $\kappa_{c}=\kappa_{\max }$. Hence, we define the statistics of $\lambda(\kappa)$, and analogously for $\lambda$, in previous equations as

$$
\begin{aligned}
\mu_{\lambda}(\kappa) & =\frac{1}{N_{r}} \sum_{k=0}^{N_{r}} \lambda(\kappa)\left(\kappa, \varphi_{k}\right) \\
\sigma_{\lambda}(\kappa) & =\left\{\frac{1}{N_{r}-1} \sum_{k=0}^{N_{r}}\left[\lambda(\kappa)\left(\kappa, \varphi_{k}\right)-\mu_{\lambda}(\kappa)\right]^{2}\right\}^{1 / 2} .
\end{aligned}
$$




\begin{tabular}{rcc}
\hline Scheme & $\mu_{\lambda} \pm 2 \sigma_{\lambda} \rightarrow \kappa_{c}=\kappa_{\max }$ & $\mu_{\lambda} \pm 2 \sigma_{\lambda} \rightarrow \kappa_{c}=\frac{2}{3} \kappa_{\max }$ \\
\hline \hline $1^{\text {st }}$-order backward & $-4.188 \pm 0.000$ & $-2.896 \pm 0.000$ \\
$2^{\text {nd }}$-order backward & $-4.200 \pm 0.000$ & $-1.845 \pm 0.000$ \\
$3^{\text {rd }}$-order backward & $-1.400 \pm 0.000$ & $-0.615 \pm 0.000$ \\
TVD scheme & $-2.960 \pm 0.074$ & $-1.465 \pm 0.044$ \\
$5^{\text {th }}$-order WENO & $-2.240 \pm 0.148$ & $-0.838 \pm 0.050$ \\
\hline $6^{\text {th }}$-order compact & $0.000 \pm 0.000$ & $0.000 \pm 0.000$ \\
DFD scheme & $6.97910^{-5} \pm 0.016$ & $-8.34510^{-6} \pm 0.008$ \\
\hline
\end{tabular}

Table 1: Normalized dissipation rate for $N_{r}=10^{5}$ realizations of a synthetic field with spectrum scaling $\kappa^{-5 / 3}$ and cutoff wavenumbers $\kappa_{c}=\kappa_{\max }$ and $\kappa_{c}=\frac{2}{3} \kappa_{\max }$.

Figure 7 shows the quantities $\lambda(\kappa)$ for the momentum conserving formulations of the TVD scheme, the $5^{\text {th }}$-order WENO scheme and the nonlinear DFD scheme. Since it concerns a linear transport equation, the nonlinear upwind schemes reduce to backward or forward difference schemes, and are therefore not considered. For the TVD scheme, the curve of $\mu_{\lambda}$ indicates a substantial mean dissipation in the entire wavenumber range, in comparison with the $2^{\text {nd }}$ and $3^{r d}$-order backward schemes. The bounding curves of the spreading, i.e. $\mu_{\lambda} \pm 2 \sigma_{\lambda}$ indicate that the dissipation can fluctuate considerately around the mean. Moreover, the upper bound of the spreading, i.e. the curve $\mu_{\lambda}+2 \sigma_{\lambda}$, indicates that in the region $\kappa<0.2 \kappa_{\max }$ anti-dissipation may occur. In other words, the nonlinearity may lead to an increase of energy for some of the very large scales. For the higher-wavenumber range, $\mu_{\lambda}$ approaches the dissipation characteristic of the $1^{\text {st }}$-order backward scheme.

For the WENO scheme, similar conclusions are drawn. The curve $\mu_{\lambda}$ also indicates a significant amount of dissipation in the entire wavenumber range, although less than the TVD scheme. The bounding curves of the spreading, i.e. $\mu_{\lambda} \pm 2 \sigma_{\lambda}$ indicate that the dissipation fluctuates substantially around the mean. The upper bound of the spreading, i.e. $\mu_{\lambda}+2 \sigma_{\lambda}$, shows that for $\kappa<0.4 \kappa_{\max }$ anti-dissipation may occur. Observe that this region is larger than for the TVD scheme. Hence, the nonlinearity may lead to an increase of energy for some large scales and medium size scales. For the higher-wavenumber range, $\mu_{\lambda}$ approaches the dissipation characteristic of the $3^{r d}$-order backward scheme, in contrast to the TVD scheme. For both the TVD scheme and the WENO scheme, the normalized dissipation predicts a significant mean dissipation on the large scales $\left(\kappa \leq 0.4 \kappa_{\max }\right)$, which is in contrast with the intended behaviour when designing these schemes. Although it is difficult to have absolute certainty, it is most likely that this should be attributed to the nonlinear interactions. For the DFD scheme, the mean dissipation $\mu_{\lambda}$ seems almost absent, and the spreading lies symmetrically around the axis. Surprisingly, the largest spreading is observed at the large scales, i.e. $\kappa<0.5 \kappa_{\max }$. Table 1 shows the values of $\lambda$ for the three nonlinear schemes, in comparison with those of linear schemes. One observes that the mean dissipation of the TVD scheme is approximately twice the dissipation of the $3^{\text {rd }}$-order backward scheme. Moreover, the standard deviation is about $2.5 \%$ of the mean dissipation. Further, the $5^{\text {th }}$ -order WENO scheme has a somewhat lower mean dissipation, but it is still significantly higher than the dissipation of the $3^{\text {rd }}$-order backward scheme. The standard deviation, however, is about $6.6 \%$ of the mean. Although the mean dissipation is not exactly zero for the DFD scheme, it is not significant. Hence, one can expect zero dissipation for $N_{r} \rightarrow \infty$. That the nonlinearity of the DFD scheme does not generate a mean dissipation, is a consequence of the purely dispersive character of the discretization (26). However, although the spreading on the dissipation is small, it is significant for the DFD scheme.

\subsubsection{Nonlinear and higher-dimensional conservation equations}

In the current work, the discussion about the conservation is restricted to the analysis of nonlinear schemes for the one-dimensional linear convection equation, since this is considered to be a simple, yet essential, case. We showed that nonlinear (central or biased) schemes can be momentum conserving but cannot be energy conserving, even in the simple case of the one-dimensional linear convection equation. Consequently, we believe that, if the conservation properties do not hold in the simple case of a linear convection equation, they will not hold for nonlinear and higherdimensional cases either. Hence, the conclusions drawn in the one-dimensional linear case, should remain valid for the discretization of higher-dimensional and nonlinear equations. Since the analysis of the conservation properties 
of nonlinear finite difference schemes is far more complex and elaborate for nonlinear and/or higher-dimensional equations, it is considered out of scope in the current paper. For further analysis of the conservation properties of linear finite difference or finite volume methods applied to the nonlinear incompressible and/or compressible NavierStokes equations, we refer to the works of Wissink [13], Morinishi et al. [14, 15], Kok [16], Subbareddy et al. [17] and Pirozzoli [18].

\section{Aliasing Errors}

A final issue that arises when applying nonlinear schemes for the discretization of derivatives, is aliasing. As shown in section 3, nonlinear schemes generate spurious scales in the entire wavenumber range due to nonlinear interactions. Moreover, the nonlinear mechanism in the discretization scheme generates spurious scales with wavenumber $\kappa>\kappa_{\max }$. Since these small scales cannot be seen on the computational grid, they are represented erroneously as aliases with a larger wavelength during simulations. This is explained further in detail.

We start the discussion by mentioning that all nonlinear schemes considered in section 2 , are in fact constructed as a spatial weighting of a series of linear finite difference flux definitions. For instance, the $1^{s t}, 2^{\text {nd }}$ and $3^{\text {rd }}$-order upwind schemes (4)-(6), are obviously a spatial weighting of backward and forward finite difference flux definitions. The central nonlinear DFD scheme (26) can be seen as a spatial weighting of a lower- and higher-order flux definition. For the TVD scheme and the WENO scheme, the weighting of the linear flux definitions may look more complicated, however, the principle remains the same. Hence, all nonlinear schemes in section 2 can be reformulated in the generic form

$$
\frac{\delta \phi}{\delta x}(x)=\frac{\sum_{k}\left(w_{k}^{+}(x) \phi_{k}^{+}(x)-w_{k}^{-}(x) \phi_{k}^{-}(x)\right)}{\Delta x},
$$

in which the spatial weighting functions are denoted by $w_{k}^{+}(x)$ and $w_{k}^{-}(x)$, whereas $\phi_{k}^{+}(x)$ and $\phi_{k}^{-}(x)$ represent the positive and negative flux definitions, which are function of the transported scalar field $u(x)$.

We continue by considering a band-limited periodic field $u(x)$, defined by expression (33) such that the energy spectrum scales with $\kappa^{-5 / 3}$ up to a cutoff wavenumber $\kappa_{c} \leq \kappa_{\max }$. Despite the fact that $u(x)$ has no Fourier modes in the wavenumber region $\kappa_{\max }-\kappa_{c}$, the weighting functions $w_{k}^{+}(x)$ and $w_{k}^{-}(x)$ are often nonlinear, non-smooth and even discontinuous functions of $u(x)$ and therefore contain wavenumbers in the entire wavenumber range $0 \leq \kappa \leq \kappa_{\max }$. Evaluation of the nonlinear expression (101) results into an approximation $\frac{\delta u}{\delta x}(x)$ for the first derivative, containing Fourier modes in the wavenumber range $0 \leq \kappa \leq \kappa_{\max }+\kappa_{c}$. Because the Fourier modes in the range $\kappa_{\max } \leq \kappa \leq \kappa_{\max }+\kappa_{c}$ cannot be represented on the computational grid, they are erroneously replaced by aliases in the wavenumber range $\kappa_{\max }-\kappa_{c} \leq \kappa \leq \kappa_{\max }$. As a consequence, they contribute to the energy level of the regular scales in that range.

The normal evaluation of the nonlinear finite difference approximation of the derivative of the field $u(x), x \in[0,2 \pi]$ in a specific node $x_{i}$ of the computational grid with $N$ nodes is given by

$$
\left.\frac{\delta u}{\delta x}\right|_{i}=\frac{\sum_{k}\left(\left.\left.w_{k}^{+}\right|_{i} f_{k}^{+}\right|_{i}-\left.\left.w_{k}^{-}\right|_{i} f_{k}^{-}\right|_{i}\right)}{\Delta x}, \quad 0 \leq i \leq N-1,
$$

in which $\left.f_{k}^{+}\right|_{i}$ and $\left.f_{k}^{-}\right|_{i}$ represent the fluxes in a node $i$. As explained before, the resulting approximation $\left.\frac{\delta u}{\delta x}\right|_{i}$ contains now aliased Fourier modes, due to the nonlinear interactions in $w_{k}^{+} f_{k}^{+}$and $w_{k}^{-} f_{k}^{-}$. In order to obtain the dealiased finite difference approximation, we first interpolate all discrete weighting functions $w_{k}^{+}$and $w_{k}^{-}$and discrete flux definitions $f_{k}^{+}$and $f_{k}^{-}$, determined on the original grid with $N$ nodes, to a computational grid with $2 N$ nodes. An exact interpolation method is used in which the interpolated field is obtained by taking the inverse Fourier transform of the spectral field $\widehat{u}(\kappa)$, padded with zeros up to the new resolution. Once the weighting functions and the discrete fluxes are interpolated, the derivative is evaluated on the fine grid with $2 N$ nodes in analogy with expression (102). Then, all Fourier modes in the range $0.5 \kappa_{\max }<\kappa \leq \kappa_{\max }$, are removed from the derivative, before sampling the derivative to the resolution of the computational grid with $N$ nodes. This way, the dealiased nonlinear finite difference approximation of the first derivative is obtained. In order to evaluate the impact of aliasing on the energy spectrum and energy balance, we make use of the NSA and the previously derived expressions for the evolution of the kinetic energy. We 

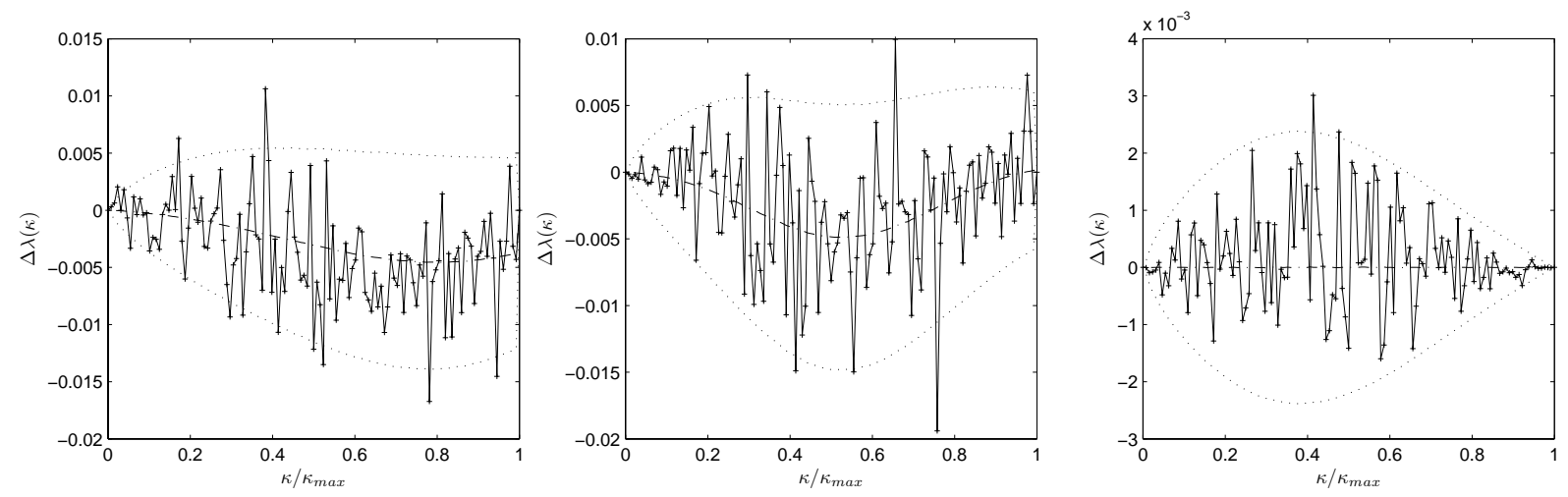

Figure 8: The aliasing contribution to the normalized dissipation rate $\Delta \lambda(\kappa)$. TVD scheme (left); $5^{\text {th }}$-order WENO scheme (center); DFD scheme (right). $\Delta \lambda(\kappa)(+) ; \mu_{\Delta \lambda}(\kappa)(-\cdot-\cdot) ; \mu_{\Delta \lambda}(\kappa) \pm 2 \sigma_{\Delta \lambda}(\kappa)(\cdots)$.

introduce the scalar fields

$$
\begin{aligned}
& \widehat{u}_{r, a}=\left.\frac{1}{i \kappa} \frac{\widehat{\delta u}}{\delta x}\right|_{a} \\
& \widehat{u}_{r, d}=\left.\frac{1}{i \kappa} \frac{\widehat{\delta u}}{\delta x}\right|_{d},
\end{aligned}
$$

which represent respectively the reconstructed fields of the aliased $\left(\widehat{u}_{r, a}\right)$ and dealiased $\left(\widehat{u}_{r, d}\right)$ nonlinear finite difference approximations. In analogy with expression (97), we define the contribution of aliasing to the spectral distribution of the normalized dissipation rate as

$$
\Delta \lambda(\kappa)=\frac{i \kappa\left[\widehat{u}\left(\widehat{u}_{r, a}^{*}-\widehat{u}_{r, d}^{*}\right)-\widehat{u}^{*}\left(\widehat{u}_{r, a}-\widehat{u}_{r, d}\right)\right]}{\int_{-\kappa_{\max }}^{\kappa_{\max }} \widehat{u} \widehat{u}^{*} d \kappa},
$$

whereas the contribution of aliasing to the total normalized dissipation rate is

$$
\Delta \lambda=\frac{\int_{-\kappa_{\max }}^{\kappa_{\max }} i \kappa\left[\widehat{u}\left(\widehat{u}_{r, a}^{*}-\widehat{u}_{r, d}^{*}\right)-\widehat{u}^{*}\left(\widehat{u}_{r, a}-\widehat{u}_{r, d}\right)\right] d \kappa}{\int_{-\kappa_{\max }}^{\kappa_{\max }} \widehat{u} \widehat{u}^{*} d \kappa} .
$$

For $\Delta \lambda<0$, the aliasing errors increase the numerical dissipation, whereas for $\Delta \lambda>0$, the aliasing errors decrease the numerical dissipation leading to an energy build up. In analogy with the NSA in section 3 , a statistical analysis is made of these quantities for $N_{r}=10^{5}$ realizations of a synthetic field with spectrum scaling $\kappa^{-5 / 3}$ and cutoff wavenumber $\kappa_{c}=\kappa_{\max }$. The statistics are defined as

$$
\begin{aligned}
\mu_{\Delta \lambda}(\kappa) & =\frac{1}{N_{r}} \sum_{k=0}^{N_{r}} \Delta \lambda(\kappa)\left(\kappa, \varphi_{k}\right) \\
\sigma_{\Delta \lambda}(\kappa) & =\left\{\frac{1}{N_{r}-1} \sum_{k=0}^{N_{r}}\left[\Delta \lambda(\kappa)\left(\kappa, \varphi_{k}\right)-\mu_{\Delta \lambda}(\kappa)\right]^{2}\right\}^{1 / 2} .
\end{aligned}
$$

Figure 8 shows the contribution of the aliasing error to the spectral distribution of the normalized dissipation rate for the TVD scheme, the $5^{\text {th }}$-order WENO scheme and the nonlinear DFD scheme. Since equation (105) is based upon the linear convection equation, the analysis of the upwind schemes is meaningless. The TVD scheme displays a negative mean $\mu_{\Delta \lambda}(\kappa)$ in the entire wavenumber range, with a minimum around $\kappa=0.8 \kappa_{\max }$. Hence, the aliasing 
error contributes in average to the numerical dissipation on all scales, but especially on the small scales in the higher wavenumber range, i.e. $\kappa \geq 0.5 \kappa_{\max }$. The spreading is quite large, and the upper bound $\mu_{\Delta \lambda}(\kappa)+2 \sigma_{\Delta \lambda}(\kappa)$ indicates that a negative contribution to the dissipation (anti-dissipation) can occur for all Fourier modes, resulting in a reduced total numerical dissipation, and thus a reduced decrease of kinetic energy. Evaluation of the mean total normalized dissipation rate, learns that $\mu_{\Delta \lambda}=-0.68$, whereas $\sigma_{\Delta \lambda}=0.13$. Hence, the aliasing errors contribute for approximately $23 \%$ to the mean numerical dissipation of the TVD scheme (see Table 1), which is substantial. Also the $5^{\text {th }}$-order WENO scheme displays a negative $\mu_{\Delta \lambda}(\kappa)$ in the entire wavenumber range, with a minimum around $\kappa=0.5 \kappa_{\max }$. Althoug, the aliasing error contributes in average to the numerical dissipation on all scales, it affects most the medium size scales around $\kappa \approx 0.5 \kappa_{\max }$. Just like for the TVD scheme, the spreading is substantial, and the upper bound $\mu_{\Delta \lambda}(\kappa)+2 \sigma_{\Delta \lambda}(\kappa)$ indicates that negative contributions to the dissipation can occur due to aliasing. Evaluation of the mean total normalized dissipation rate, learns that $\mu_{\Delta \lambda}=-0.62$, whereas $\sigma_{\Delta \lambda}=0.098$. Hence, the aliasing errors contribute for approximately $28 \%$ to the mean numerical dissipation of the WENO scheme, which is more than for the TVD scheme. For the nonlinear DFD scheme, one observes, surprisingly, that the mean contribution of the aliasing error to the dissipation is almost zero in the entire wavenumber range. The spreading shows that aliasing errors do occur, but contribute either positively or negatively. The statistics on the total normalized dissipation rate learn that $\mu_{\Delta \lambda}=1 e-5$, which is insignificant. The standard deviation is $\sigma_{\Delta \lambda}=0.019$. We emphasize again that the previous analysis of aliasing errors is only valid for a single time-step advancement, and does not take into account the build up of aliasing errors during time-advancement in a real simulation. Indeed, repeated application of nonlinear schemes in real simulations may lead to a larger contribution of the aliasing errors.

\section{Numerical investigation}

In order to study the behaviour of nonlinear finite difference schemes in the context of long-time integration in a practical simulation, and in order to verify the conclusions of the Nonlinear Spectral Analysis, two elementary numerical experiments are performed. First, the one-dimensional linear convection equation is considered, in which a particular field is transported through a periodic domain. Further, the one-dimensional viscous Burgers' equation is considered in which an energy cascade is maintained in equilibrium by applying a specific energy-conserving forcing. This way, the kinetic energy, dissipated by the viscous terms, is injected again in the large scales. In both cases, the generation and spectral distribution of spurious scales is analyzed, as well as the evolution of the kinetic energy.

\subsection{One-dimensional linear convection equation.}

The one-dimensional convective transport of a scalar $u(x), x \in[0,2 \pi]$ on a computational grid with uniform grid spacing $\Delta x$, is described by the discrete convection equation

$$
\frac{\partial u}{\partial t}(x, t)+v \frac{\delta u}{\delta x}(x, t)=0,
$$

in which $v$ denotes the constant transporting velocity. The initial field $u(x, t=0)$ is determined in Fourier space by the relation

$$
\widehat{u}(\kappa)= \begin{cases} \pm \sqrt{U_{0}} \frac{\left(1+i \tan \left(\varphi_{u}(\kappa)\right)\right)}{\sqrt{1+\tan ^{2}\left(\varphi_{u}(\kappa)\right)}} \kappa^{\alpha / 2} & 0<\kappa \leq \kappa_{c} \\ 0+i 0 & \kappa_{c}<\kappa \leq \kappa_{\max }\end{cases}
$$

in which we chose $\alpha=-5 / 3$ and $\kappa_{c}=2 \kappa_{\max } / 3$. The computational grid has a resolution of $N=1024$ nodes, or $\kappa_{\max }=512$ Fourier modes. The phase function $\varphi_{u}(\kappa)$ is uniformly distributed in the interval $[-\pi, \pi]$, whereas the mean of $u(x)$ and the magnitude $U_{0}$ are chosen arbitrarily. For the time stepping, the standard 6-stage low-storage Runge-Kutta method is selected, leading to following algorithm for stage $j$

$$
u^{t+\frac{j}{6}}=u^{t}-\alpha_{j} \Delta t\left[v \frac{\delta u}{\delta x}(x, t)\right]^{t+\frac{j-1}{6}}, \quad j=1,2, \ldots, 6
$$


in which the coefficients are given by

$$
\alpha_{j}=\frac{1}{7-j}, \quad j=1,2, \ldots, 6 .
$$

The transporting velocity is chosen $v=\pi$. The time step is set to $\Delta t=1 e-6$, which is sufficiently small to ensure that the numerical dispersion and dissipation of the Runge-Kutta method are negligible $(<1 e-15)$ and do not interfere with the dispersion and dissipation of the spatial discretization. The CFL-number lies in the order $10^{-4}$, which is very small. Further, the initial scalar field $u(x, t=0)$ is transported 1 cycle through the periodic domain, such that the total number of time steps is determined as $n_{t}=2 \pi / v \Delta t=2 e 6$, and $t_{\text {end }}=n_{t} \Delta t=2$.

The TVD scheme (8), the $5^{\text {th }}$-order WENO scheme (11), and the DFD scheme (26), are adopted for the discretization of the convective term. Since the convection equation is linear, the nonlinear upwind schemes (4), (5) and (6), reduce to the standard backward finite difference schemes. Figure 9 show the relative loss in kinetic energy $\Delta k(t)$ and the normalized total dissipation rate $\lambda(t)$, defined respectively as

$$
\begin{aligned}
\Delta k(t) & =\frac{k(t)-k_{0}}{k_{0}} \\
\lambda(t) & =\frac{1}{v k_{0}} \frac{k(t)-k(t-\Delta t)}{\Delta t},
\end{aligned}
$$

for the TVD scheme, the $5^{\text {th }}$-order WENO scheme and the DFD scheme. For the TVD scheme, the numerical dissipation $\lambda(t)$ in the initial stages of the simulation, is close to that of the $2^{\text {nd }}$-order backward scheme. Note that in this region, the shape of the energy spectrum is not much different of that of the initial field. As the simulation proceeds, the numerical dissipation of the upwind and the TVD schemes leads to more and more smoothing of the solution, damping substantially the kinetic energy of small scales at the tail of the energy spectrum. However, the numerical dissipation of the TVD scheme seems to decreases slower than that of the $2^{\text {nd }}$-order backward scheme. In a way, this is contra-intuitive since TVD schemes are expected to become less dissipative for very smooth fields.

The numerical dissipation $\lambda(t)$ of the $5^{\text {th }}$-order WENO scheme in the initial stages of the simulation, is close to that of the $3^{\text {rd }}$-order backward scheme. As the simulation proceeds, the numerical dissipation first seems to decrease at a slower rate than that of the $3^{r d}$-order scheme. However, as soon as the solution smoothens, the dissipation rate decreases $\lambda(t)$ more rapidly, resulting in a smaller numerical dissipation than that of the $3^{\text {rd }}$-order backward scheme at the end of the simulation. For the nonlinear DFD scheme, the numerical dissipation rate $\lambda(t)$ fluctuates substantially around a small positive time-average, during the entire simulation time. These fluctuations seem to increase as the simulation advances in time. Although the total kinetic energy alternately increases and decreases in the initial stages of the simulation, the positive average value of $\lambda(t)$, results in a systematic increase of kinetic energy as the simulation proceeds. Hence, in contrast to the theoretical predictions obtained by the Nonlinear Spectral Analysis, the nonlinear DFD scheme leads to a non-zero mean dissipation component.

Figure 10 displays the relative error on the energy spectrum $\Delta E_{u}(\kappa, t)$ and the energy spectrum of the error $E_{\Delta_{u}}(\kappa, t)$, defined as

$$
\begin{aligned}
\Delta E_{u}(\kappa, t) & =\frac{\widehat{u} \widehat{u}^{*}-\widehat{u}_{r} \widehat{u}_{r}^{*}}{\int_{-\kappa_{\max }}^{\kappa_{\max }} \widehat{u} \widehat{u}^{*} d \kappa} \\
E_{\Delta_{u}}(\kappa, t) & =\frac{\left(\widehat{u}^{*}-\widehat{u}_{r}\right)\left(\widehat{u}^{*}\right)}{\int_{-\kappa_{\max }}^{\kappa_{\max }} \widehat{u} \widehat{u}^{*} d \kappa},
\end{aligned}
$$

in which $u_{r}$ denotes the resolved finite difference solution, and $u$ represents the exact spectral solution. Whereas $\Delta E_{u}(\kappa, t)$ describes only the error on the amplitude of the different scales in the solution, $E_{\Delta_{u}}(\kappa, t)$ contains information about both the amplitude and the phase of the different scales in the solution. The plots of $\Delta E_{u}(\kappa, t)$ at $t / t_{\text {end }}=1$, i.e. after one cycle through the domain, show that the errors on the energy content of the large scales $\kappa<0.1 \kappa_{\max }$ are quite substantial for all three nonlinear schemes in comparison with the linear schemes of order 2 and more. Note that the linear central finite difference approximations, used for comparison of the DFD scheme, do not generate errors on 

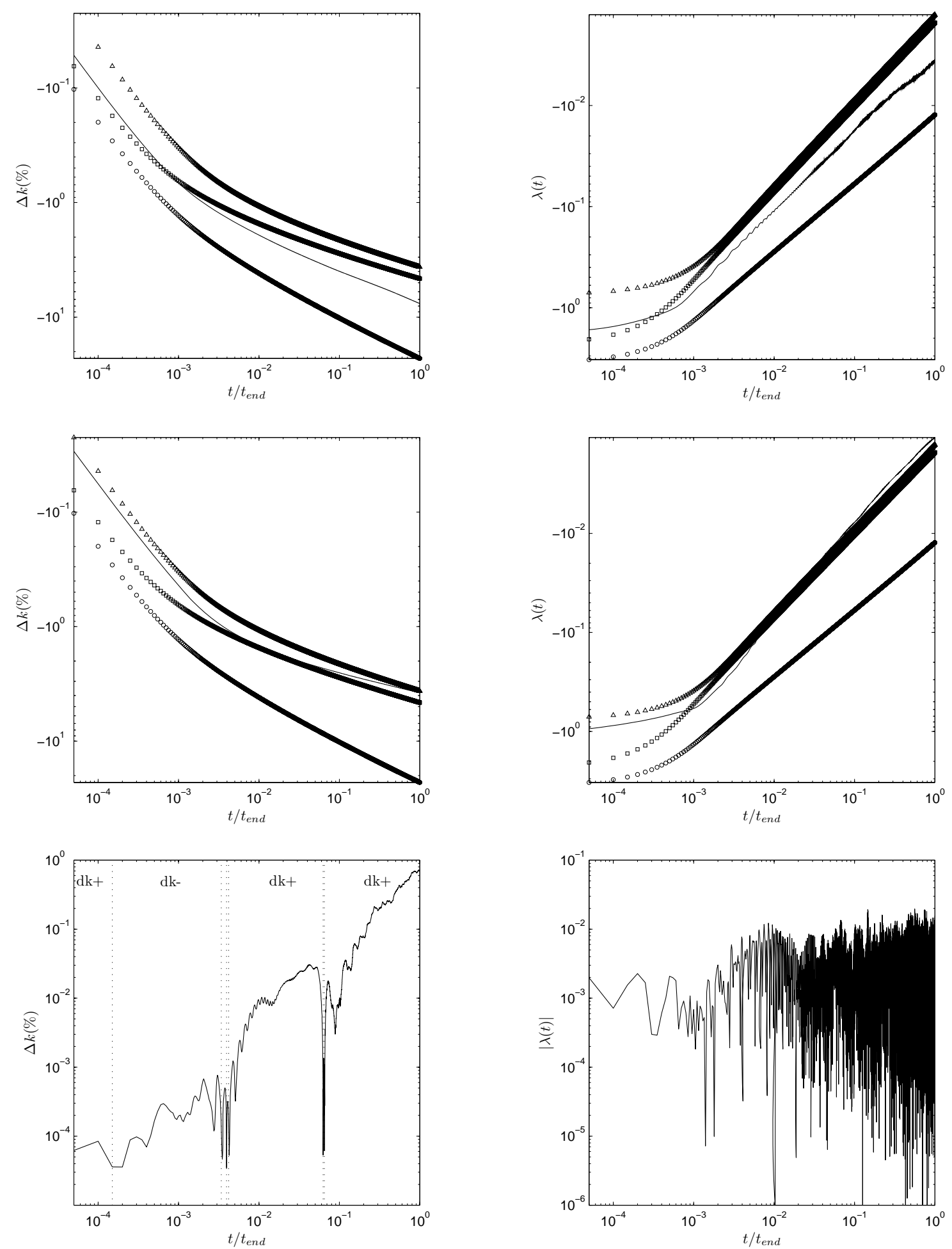

Figure 9: Kinetic energy and dissipation: $\Delta k(t)(\%)\left(1^{\text {st }}\right.$ column); $\lambda(t)\left(2^{\text {nd }}\right.$ column). TVD scheme ( $1^{\text {st }}$ row); $5^{\text {th }}$-order WENO scheme $\left(2^{\text {nd }}\right.$ row); DFD scheme ( $3^{\text {rd }}$ row). Symbols for the TVD and WENO schemes: $1^{\text {st }}$-order backward (o); $2^{\text {nd }}$-order backward ( $\square$ ); $3^{\text {rd }}$-order backward $(\Delta)$. Scheme of interest ( - $)$. Remark that for the DFD scheme, zones of positive and negative $\Delta k(t)(\%)$, denoted by $d k-$ and $d k+$, are separated by the dotted line $(\cdots)$. 
the energy spectrum, since they are skew-symmetric and thus exactly conserve the kinetic energy. The TVD scheme displays the largest errors that seem to scale as $\kappa^{0}, \forall \kappa<0.05 \kappa_{\max }$. The WENO scheme displays a similar $\kappa^{0}$-error plateau for $\kappa<0.2 \kappa_{\max }$, although it is lower in value than the TVD scheme. The DFD scheme, which is the most accurate, shows an error plateau for $\kappa<0.5 \kappa_{\max }$. Both $\Delta E_{u}(\kappa, t)$ and $E_{\Delta_{u}}(\kappa, t)$ at $t / t_{\text {end }}=1$, show that the TVD scheme and the WENO scheme scale as a $1^{s t}$-order accurate scheme for $\kappa<0.1 \kappa_{\text {max }}$. Hence, the TVD does not maintain the designed $2^{\text {nd }}$-order accuracy for the large and smooth scales in the solution. Also the WENO scheme does not maintain the $5^{\text {th }}$-order accuracy, intended by the design. The DFD scheme scales as a $1^{\text {st }}$-order accurate scheme for $\kappa<0.5 \kappa_{\max }$, although the accuracy remains better than the $2^{\text {nd }}$-order central scheme. Since, no biased stencils can occur in case of the DFD scheme, the $1^{s t}$-order behaviour can only be attributed to the nonlinearity. For $\kappa_{c} \leq \kappa \leq \kappa_{\max }$, spurious Fourier modes are observed for all three schemes although their energy decreases rapidly with increasing wavenumber. For the WENO scheme, these spurious scales are lower than for the other nonlinear schemes.

\subsection{One-dimensional Burgers' equation.}

The one-dimensional forced Burgers' equation for a velocity field $u(x), x \in[0,2 \pi]$, is described by the analytical equation

$$
\frac{\partial u}{\partial t}+\frac{1}{2} \frac{\partial u u}{\partial x}=\frac{1}{\operatorname{Re}} \frac{\partial^{2} u}{\partial x^{2}}+f
$$

The discrete equation on a computational grid with uniform grid spacing $\Delta x$ is then defined as,

$$
\frac{\partial u}{\partial t}+\frac{u}{3} \frac{\delta u}{\delta x}+\frac{1}{3} \frac{\delta u^{2}}{\delta x}=\frac{1}{\operatorname{Re}} \frac{\delta^{2} u}{\delta x^{2}}+f
$$

The skew-symmetric formulation for the nonlinear term is adopted, i.e. a combination of the advective and divergence formulations of the nonlinear term, since this formulation ensures the kinetic energy conservation of the nonlinear term for all linear central discretization schemes [19]. As a consequence, for these schemes, only the viscous term and the forcing term contribute to the dissipation. Appendix A describes the discretization of the skew-symmetric term, using the nonlinear upwind schemes, the TVD scheme, the $5^{\text {th }}$-order WENO scheme and DFD scheme. The initial scalar field $u(x, t=0)$ is determined in Fourier space by the relation

$$
\widehat{u}(\kappa)= \begin{cases} \pm \sqrt{U_{0}} \frac{\left(1+i \tan \left(\varphi_{u}(\kappa)\right)\right)}{\sqrt{1+\tan ^{2}\left(\varphi_{u}(\kappa)\right)}} \kappa^{\alpha / 2} e^{\beta \kappa / 2} & 0<\kappa \leq \kappa_{c} \\ 0+i 0 & \kappa_{c}<\kappa \leq \kappa_{\max }\end{cases}
$$

in which $\alpha=-2$ is selected, corresponding to the natural scaling of Burgers'solution. We chose a computational grid with a resolution of $N=1024$ nodes, or $\kappa_{\max }=512$ Fourier modes. The phase function $\varphi_{u}$ is uniformly distributed in the interval $[-\pi, \pi]$, whereas the magnitude $U_{0}$ is chosen such that $\max (u)-\min (u)=2$ and the mean velocity $\bar{u}=\pi / 8$. This ensures that the initial field has positive and negative values. The dissipation range of the scalar field is determined by the damping parameter $\beta$. This parameter is set to $\beta=0.1$, such that all scales in the solution are well resolved, i.e. the energy in the smallest scales is in the order of the machine precision. The cutoff wavenumber is then safely set to $\kappa_{c}=\kappa_{\max }$. Finally, the Reynolds number is set to $\mathrm{Re}=100$. For the time stepping, again the standard 6-stage low-storage Runge-Kutta method is selected, leading to following algorithm for stage $j$

$$
u^{t+\frac{j}{6}}=u^{t}-\alpha_{j} \Delta t\left[\frac{u}{3} \frac{\delta u}{\delta x}+\frac{1}{3} \frac{\delta u^{2}}{\delta x}-\frac{1}{\operatorname{Re}} \frac{\delta^{2} u}{\delta x^{2}}-f\right]^{t+\frac{j-1}{6}}, \quad j=1,2, \ldots, 6 .
$$

in which the coefficients are given by

$$
\alpha_{j}=\frac{1}{7-j}, \quad j=1,2, \ldots, 6 .
$$

The time step is set to $\Delta t=1 e-7$, which is sufficiently small to ensure that the numerical dispersion and dissipation of the Runge-Kutta method are negligible and do not interfere with the dispersion and dissipation of the spatial 

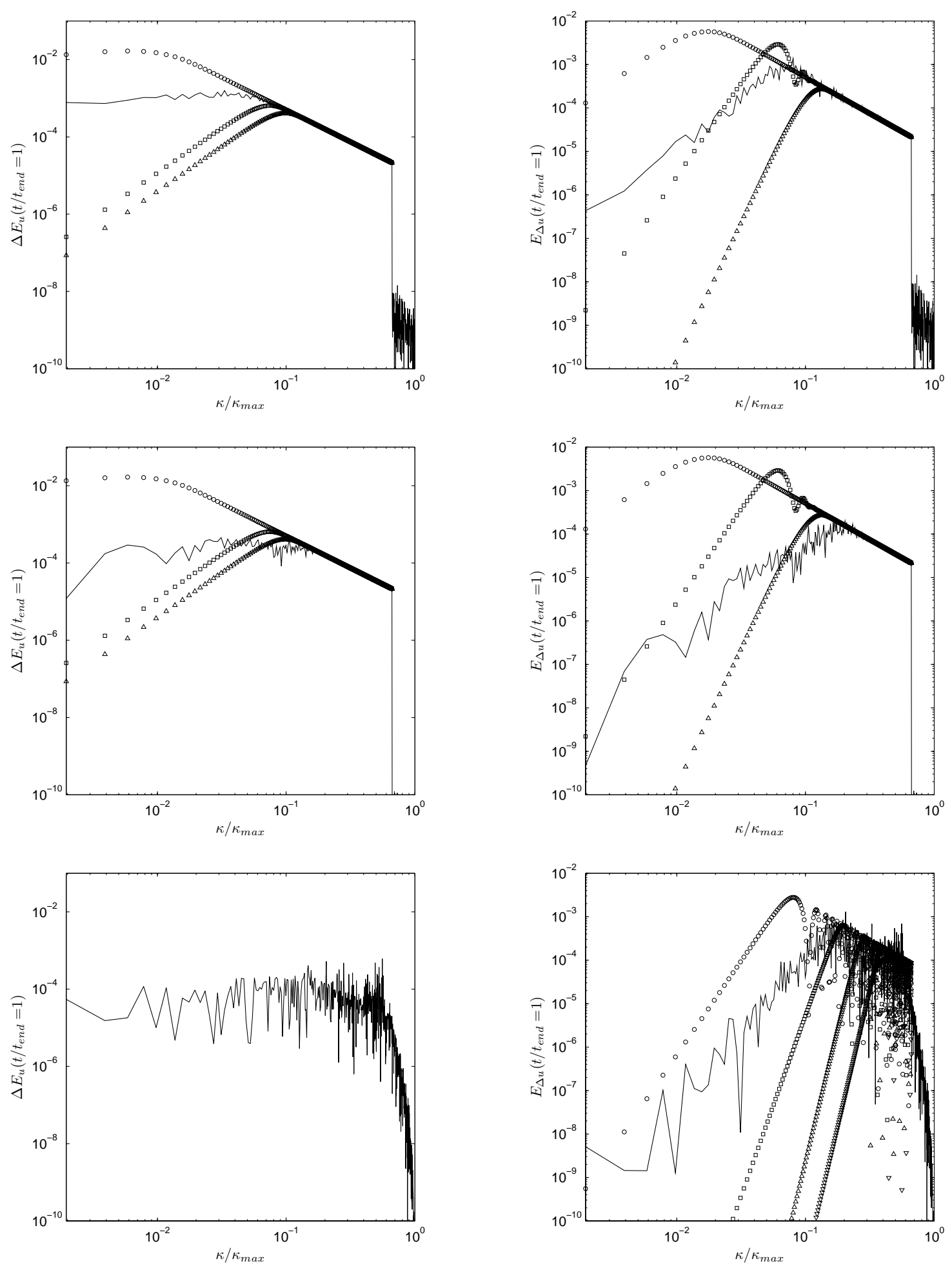

Figure 10: Spectral error distributions: $\Delta E_{u}(\kappa, t) @ t / t_{\text {end }}=1\left(1^{\text {st }}\right.$ column $) ; E_{\Delta_{u}}(\kappa, t) @ t / t_{\text {end }}=1\left(2^{\text {nd }}\right.$ column $)$ TVD scheme $\left(1^{\text {st }}\right.$ row $) ; 5^{\text {th }}$ -order WENO scheme ( $2^{\text {nd }}$ row); DFD scheme ( $3^{\text {rd }}$ row). Symbols for the TVD and WENO schemes: $1^{\text {st }}$-order upwind (o); $2^{\text {nd }}$-order upwind $(\square) ; 3^{\text {rd }}$-order upwind $(\triangle)$. Symbols for the DFD-scheme: $2^{\text {nd }}$-order central $(\circ) ; 4^{\text {th }}$-order central $(\square) ; 6^{\text {th }}$-order tridiagonal compact $(\Delta)$. Scheme of interest (-). 

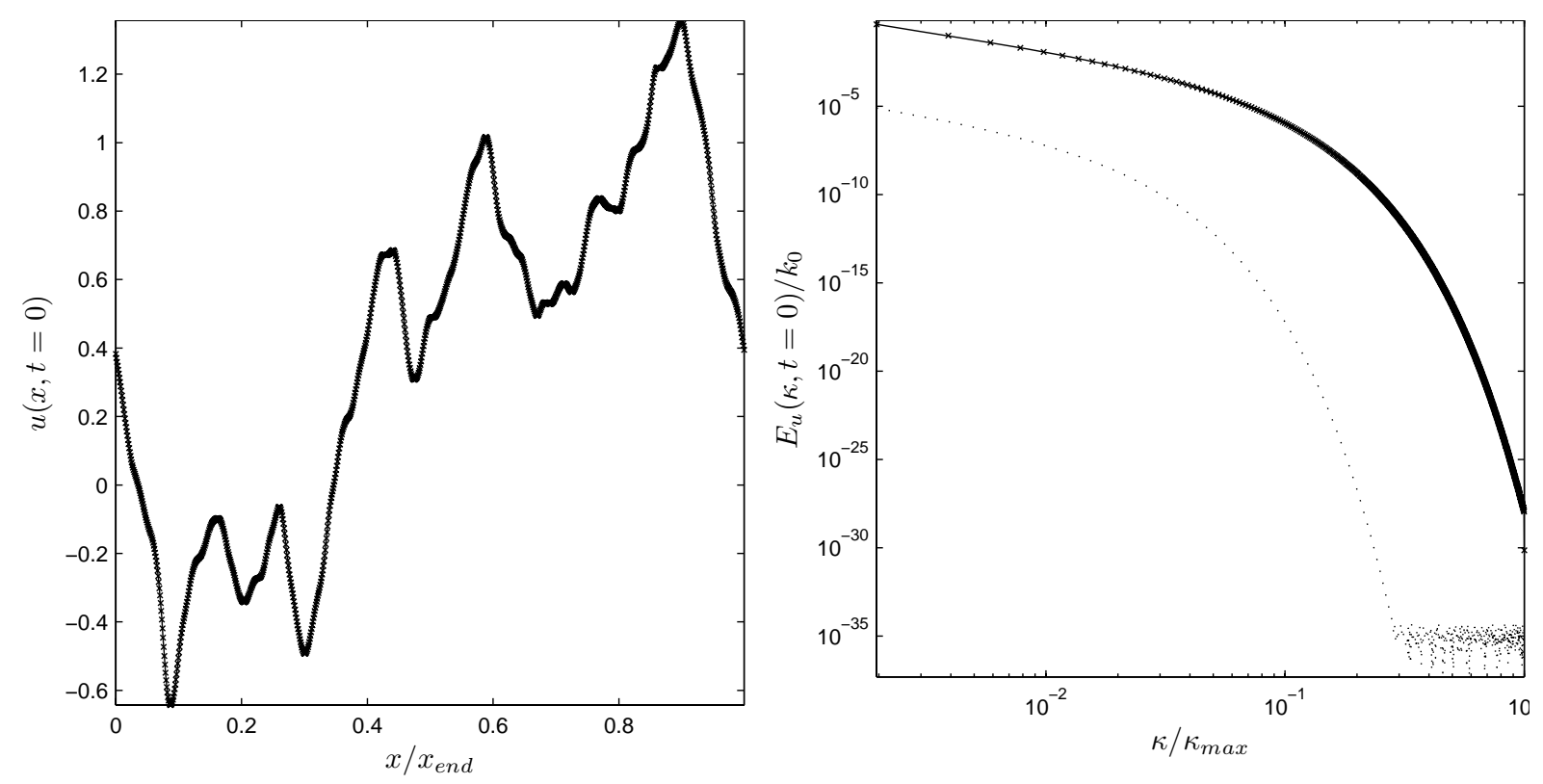

Figure 11: Initial scalar field $u(x, t=0): u(x, t=0)$ (left); Energy Spectrum $E_{u}(\kappa, t=0)$ (right). Energy spectrum of the forcing $\Delta u_{f}(\kappa, t=0)$ is shown $(\cdots)$.

discretization. The CFL-number is very small. In order to transport the initial scalar field $u(x, t=0)$ it would take approximately $n_{t}=2 \pi / \bar{u} \Delta t=16 e 7$ time steps for 1 cycle through the periodic domain. Because this would take a very long time to calculate, the initial field is advanced only through a fraction of the domain, however, large enough to conclude about the evolution of the kinetic energy. Figure 11 displays the initial scalar field, in combination with its energy spectrum. Obviously, the smallest scales in this field have only little energy, which implies that all scales are represented well on the computational grid.

The purpose of the forcing term, is to compensate for the energy drain due to the viscous dissipation. More specifically, the forcing is constructed such that the energy, dissipated by the viscous term at the small scales, is injected again at the large scales, such that the energy cascade is maintained and the decay of Burgers' turbulence is prevented. Hence, for Fourier spectral discretizations or linear central discretization schemes, the energy balance remains in equilibrium, i.e. no net energy is drained from or added to the solution. This approach enables us to study the impact of the discretization of the nonlinear term on the solution, and in particular, on the energy balance. In the following, we explain the construction of the forcing term. Since the purpose of the forcing term is only to compensate for the viscous energy dissipation, we consider the partially updated field $u_{v}$ at Runge-Kutta stage $j$, i.e.

$$
u_{v}^{t+\frac{j}{6}}=u^{t}+\alpha_{j} \Delta t\left[\frac{1}{\operatorname{Re}} \frac{\delta^{2} u}{\delta x^{2}}\right]^{t+\frac{j-1}{6}}+\alpha_{j} \Delta t f^{t+\frac{j-1}{6}} .
$$

Substitution of $u_{v}^{*}=u^{t}+\alpha_{j} \Delta t\left[\frac{1}{\operatorname{Re}} \frac{\delta^{2} u}{\delta x^{2}}\right]^{t+\frac{j-1}{6}}$ and defining the force as

$$
f^{t+\frac{j-1}{6}}=a \frac{\Delta u_{f}}{\alpha_{j} \Delta t}
$$

yields

$$
u_{v}^{t+\frac{j}{6}}=u_{v}^{*}+a \Delta u_{f} .
$$

The parameter $a$ determines the magnitude of the forcing, and the scalar field $\Delta u_{f}$ is redefined each time step according to prescription (119). The mean of $\Delta u_{f}$ is chosen zero and $U_{0}=1$, whereas the damping parameter $\beta$ is chosen 4 
times that of the initial field. This restricts the forcing to mostly the large scales. Figure 11 gives an illustration of the characteristic shape of the energy spectrum of the forcing $\Delta u_{f}$.

The conservation of kinetic energy at each Runge-Kutta step, is now expressed by the relation

$$
\int_{0}^{2 \pi} \frac{\left(u_{v}^{t+\frac{j}{6}}\right)^{2}}{2} d x=\int_{0}^{2 \pi} \frac{\left(u^{t}\right)^{2}}{2} d x
$$

Evaluating the left hand side in previous expression as

$$
\begin{aligned}
\int_{0}^{2 \pi} \frac{\left(u_{v}^{t+\frac{j}{6}}\right)^{2}}{2} d x & =\int_{0}^{2 \pi} \frac{\left(u_{v}^{*}+a \Delta u_{f}\right)^{2}}{2} d x \\
& =a^{2} \int_{0}^{2 \pi} \frac{\left(\Delta u_{f}\right)^{2}}{2} d x+a \int_{0}^{2 \pi} u_{v}^{*} \Delta u_{f} d x+\int_{0}^{2 \pi} \frac{\left(u_{v}^{*}\right)^{2}}{2} d x
\end{aligned}
$$

one obtains the condition

$$
a^{2} \underbrace{\int_{0}^{2 \pi} \frac{\left(\Delta u_{f}\right)^{2}}{2} d x}_{T_{1}}+a \underbrace{\int_{0}^{2 \pi} u_{v}^{*} \Delta u_{f} d x}_{T_{2}}+\underbrace{\int_{0}^{2 \pi} \frac{\left(u_{v}^{*}\right)^{2}-\left(u^{t}\right)^{2}}{2} d x}_{T_{3}}=0
$$

which is a quadratic equation in the forcing magnitude $a$. The magnitude $a$ is then readily obtained by finding the minimum root, i.e.

$$
a=\min \left(\frac{-T_{2} \pm \sqrt{T_{2}^{2}-4 T_{1} T_{3}}}{2 T_{1}}\right) .
$$

Once the forcing magnitude is known, one can update the scalar field as

$$
u^{t+\frac{j}{6}}=u^{t}-\alpha_{j} \Delta t\left[\frac{u}{3} \frac{\delta u}{\delta x}+\frac{1}{3} \frac{\delta u^{2}}{\delta x}-\frac{1}{\operatorname{Re}} \frac{\delta^{2} u}{\delta x^{2}}-a \frac{\Delta u_{f}}{\alpha_{j} \Delta t}\right]^{t+\frac{j-1}{6}}, \quad j=1,2, \ldots, 6
$$

The update contains now the same amount of energy as the field $u^{t}$, if the discretization of the nonlinear term is energy conserving.

Figure 12 displays the decay of kinetic energy $\Delta k(t)$, defined by expression (113). The results for the $1^{\text {st }}-, 2^{\text {nd }}$ - and $3^{r d}$-order upwind schemes, the TVD scheme and the $5^{\text {th }}$-order WENO scheme are shown in the left panel whereas the results for the nonlinear DFD scheme are shown in the right panel. For the three upwind schemes, the TVD scheme and the WENO scheme, the kinetic energy decreases ( $|\Delta k|$ increases) as expected, since the numerical dissipation is dominant. Around $t / t_{\text {end }}=0.3$, a steep increase of the dissipation is observed due to the merging of small shock fronts to a larger shock front. Due to the merging, the energy in the small scales increases leading to a larger numerical dissipation. The TVD scheme follows very closely the characteristic of the $2^{\text {nd }}$-order upwind scheme during the entire simulation. This is due to the specific choice of the limiter function, which makes that most of the time, the $2^{\text {nd }}$-order upwind scheme is engaged. The $5^{\text {th }}$-order WENO scheme displays much lower levels of numerical dissipation for $t / t_{\text {end }} \leq 0.3$, in comparison with the upwind schemes and the TVD schemes. This corresponds with the expected behaviour of the $5^{\text {th }}$-order WENO scheme in smooth conditions. However, as soon as the small shock fronts merge, the numerical dissipation increases much more than for the other schemes, such that the kinetic energy decreases rapidly to the same level of the $3^{\text {rd }}$-order upwind scheme. In general, one may conclude that the 

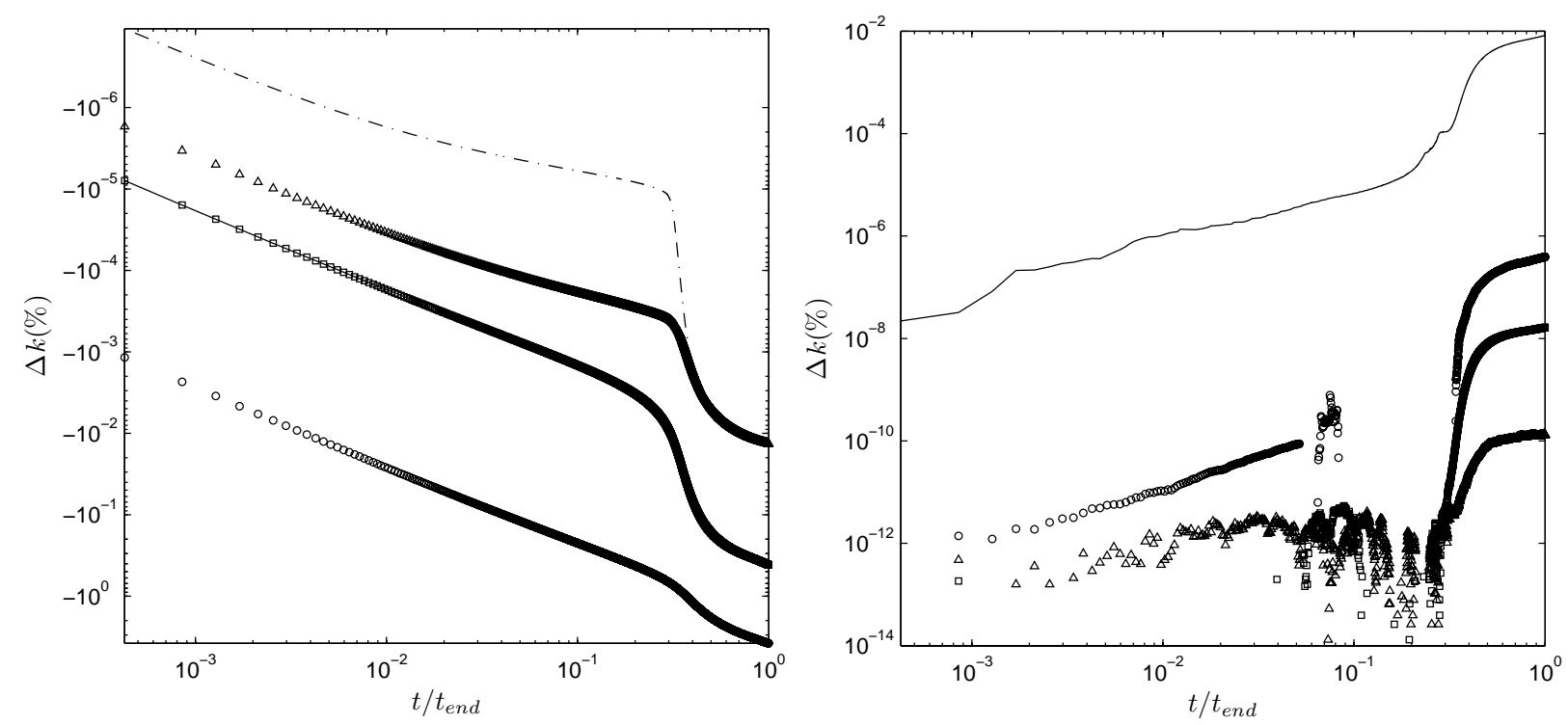

Figure 12: Decay of kinetic energy $\Delta k(t)$ : Upwind schemes, TVD scheme and WENO scheme (left): $1^{s t}$-order upwind (o); $2^{\text {nd }}$-order upwind $(\square) ; 3^{\text {rd }}$-order upwind $(\triangle)$; TVD scheme $(-) ; 5^{\text {th }}$-order WENO scheme (-.-.). DFD-scheme (right): $2^{\text {nd }}$-order central (o); $4^{\text {th }}$-order central $(\square) ; 6^{\text {th }}$-order tridiagonal compact $(\triangle)$; DFD scheme $(-)$.

nonlinearity in the nonlinear schemes, does not lead to unexpected behaviour of the results. Indeed, the impact of the nonlinear mechanism seems of minor importance in comparison with the dominant numerical dissipation.

For the DFD scheme and other central schemes, the picture is a little different. As expected, $\Delta k(t)$ is very small for the $2^{\text {nd }}$ - and $4^{\text {th }}$-order central scheme as well as for the $6^{\text {th }}$-order compact scheme. The only contribution to the dissipation is due to the Runge-Kutta time-stepping, which remains very low because of the small time step, but nevertheless leads to a small loss of energy conservation. For the DFD-scheme, one observes that $\Delta k(t)$ is positive and significantly higher, i.e. almost 4 orders of magnitude, than for the other schemes. Hence, the kinetic energy systematically increases due to the nonlinear mechanism, despite the small dissipation of the Runge-Kutta time stepping. Around $t / t_{\text {end }}=0.3$, the steep increase of $\Delta k(t)$ has the same cause as explained for the TVD scheme. Due to the merging of small shocks, the energy in the small scales increases, and because central schemes contain no numerical dissipation, this extra energy is not dissipated fast enough by the molecular viscosity, resulting in aliasing and an increase of total kinetic energy.

The error on the energy spectrum $\Delta E_{u}$ and the energy spectrum of the spatial error $E_{\Delta_{u}}$, respectively defined by expressions (115) and (116), are shown in figure 13. For the upwind schemes, the TVD scheme and the $5^{\text {th }}$ order WENO scheme, both $\Delta E_{u}$ and $E_{\Delta_{u}}$ seem to indicate error levels that lie in the same order of magnitude for $\kappa / \kappa_{\max } \rightarrow 0$. This seems to indicate that for the largest, and thus smoothest scales, the expected asymptotic accuracy is not maintained by the solution. However, in the wavenumber region, $0.01 \leq \kappa / \kappa_{\max } \leq 0.25$, one clearly notices that the $1^{\text {st }}$-order upwind scheme generates the largest errors, followed by the TVD scheme and the $2^{\text {nd }}$-order upwind scheme, who have almost identical errors. Surprisingly, the $3^{\text {rd }}$-order upwind scheme does not perform much better than the $2^{\text {nd }}$-order upwind scheme. The WENO scheme seems to have the smallest errors in that wavenumber range. Interesting is the behaviour of the error spectrum in the high-wavenumber range, i.e. $\kappa>0.5 \kappa_{\max }$. Obviously, one notices there the creation of spurious scales due to nonlinearity for all nonlinear schemes. The $1^{\text {st }}$-order upwind scheme, generates the most energetic spurious scales, followed by respectively the $5^{\text {th }}$-order WENO scheme, the TVD scheme and the $2^{\text {nd }}$ - and $3^{\text {rd }}$-order upwind schemes. The tail of the error spectrum of the TVD scheme displays a more erratic behaviour than those of the other schemes.

For the DFD scheme, the results of $\Delta E_{u}$ and $E_{\Delta_{u}}$ are close to those of the $2^{\text {nd }}$-order central scheme in the low 

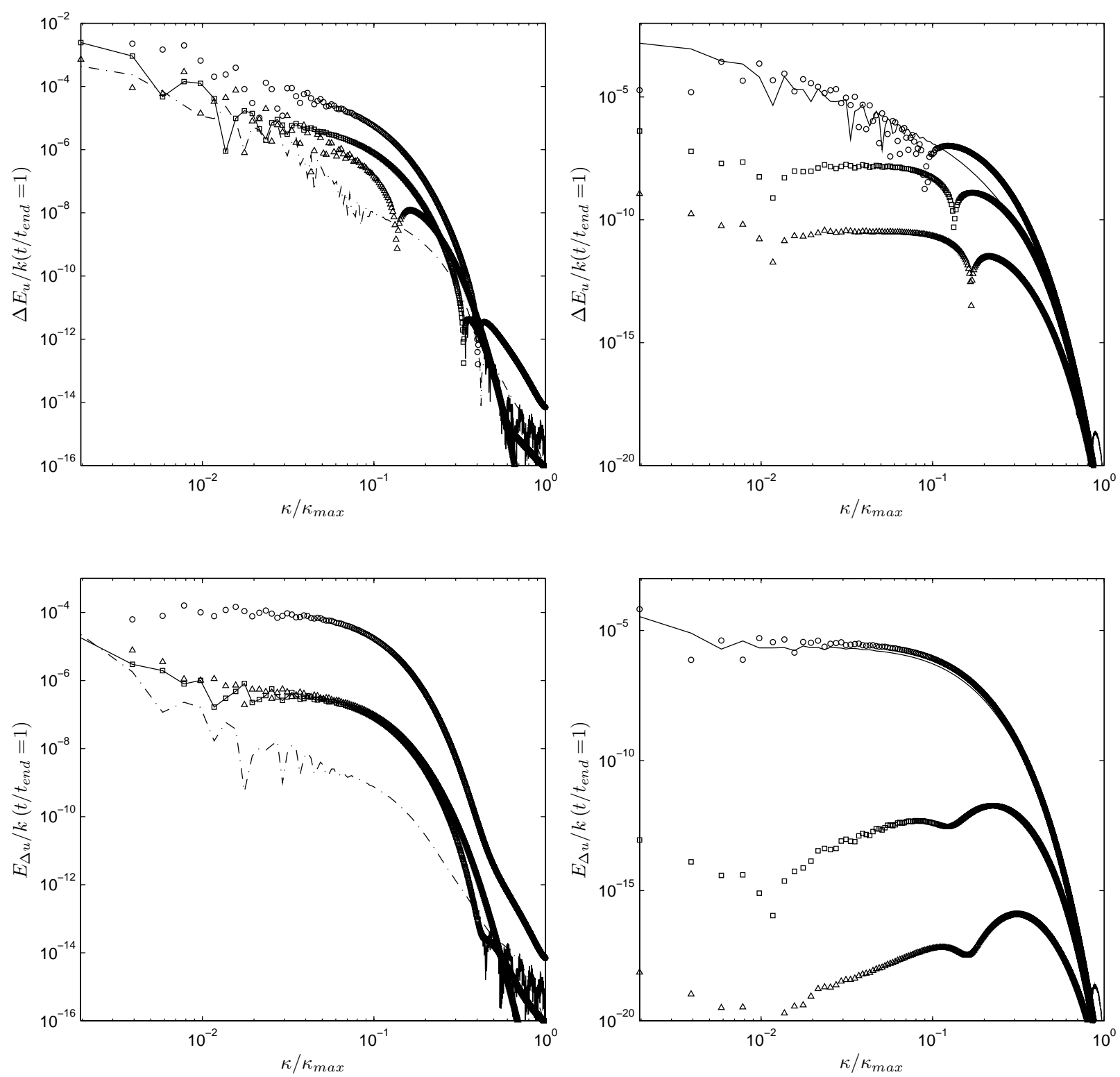

Figure 13: Spectral error distributions: $\Delta E_{u}(\kappa, t) @ t / t_{\text {end }}=1\left(1^{\text {st }}\right.$ row $) ; E_{\Delta_{u}}(\kappa, t) @ t / t_{\text {end }}=1$ ( $2^{\text {nd }}$ row $)$. Upwind schemes, TVD scheme and WENO scheme (left): $1^{\text {st }}$-order upwind (o); $2^{\text {nd }}$-order upwind ( $\square$ ); $3^{\text {rd }}$-order upwind $(\triangle)$ ) TVD scheme $(-)$ ); $5^{\text {th }}$-order WENO scheme (-.-.). DFD-scheme (right): $2^{\text {nd }}$-order central (o); $4^{\text {th }}$-order central $(\square) ; 6^{\text {th }}$-order tridiagonal compact $(\triangle)$; DFD scheme $(-)$. 
wavenumber range. Obviously, the nonlinear mechanism in the DFD scheme leads to a significant loss of accuracy on the largest scales. Once $\kappa / \kappa_{\max }>0.1$, the DFD-error decreases, reaching a comparable accuracy as that of the $4^{\text {th }}$ -order central scheme for $\Delta E_{u}$, but apparently not for $E_{\Delta_{u}}$. The quality of the $6^{\text {th }}$-order Padé scheme is never reached, despite the expectations based on the modified wavenumber for a single wave [6]. In the high wavenumber range, one notices a small bump in the energy spectrum, due to the spurious scales, generated by the DFD scheme.

We conclude that although the upwind schemes, the TVD scheme and the WENO scheme, do not display unexpected behaviour due to the dominant numerical dissipation, the nonlinear mechanism disturbs the accuracy of the very large scales in the field. Since the nonlinear DFD scheme is not dissipative, the nonlinear mechanism is dominant here, leading to a significant loss of accuracy in the entire wavenumber range, in comparison with the theoretical expectations. Moreover, all nonlinear schemes generate spurious modes in the high-wavenumber range, although these scales are damped primarily by viscosity. As a concluding remark, we emphasize that in the current simulations, all scales are resolved quite well at all times, such that the energy at the small scales is very low. Hence, this is comparable with a Direct Numerical Simulation. In case the smallest scales are not resolved, comparable to what happens in a Large Eddy Simulation, the smallest resolved scales can contain a significant amount of energy. Hence, the impact of the nonlinear mechanisms in the schemes under investigation, will be much larger in an LES-like simulation, than in a DNS-like simulation. We note that repeating this analysis in the context of Large-Eddy Simulation would be difficult and more cumbersome, since some subgrid modeling would be required.

\section{Conclusions}

Following the motivations of Pirozzoli [1] to provide better understanding about the properties of nonlinear finite difference operators, the present work is dedicated to the detailed theoretical analysis of the spectral properties and the conservation properties of a representative selection of nonlinear finite difference discretizations, including upwind schemes, a standard TVD scheme, the $5^{\text {th }}$-order WENO scheme and the nonlinear DFD scheme. We proposed a new Nonlinear Spectral Analysis (NSA) in order to study the statistical behaviour of e.g. the modified wavenumber of a nonlinear finite difference operator, and we compared it with the quasi-linear ADR-method, proposed Pirozzoli [1]. Further, we derived analytically the necessary conditions for nonlinear schemes in order to satisfy the global and local conservation of momentum and kinetic energy. Moreover, we investigated the effect of aliasing errors, that occur due to the nonlinear interactions. Finally, we verified the theoretical observations by the one-dimensional simulation of the linear convection equation and the nonlinear Burgers' equation with energy conserving forcing. The most important conclusions in this work are enlisted hereafter.

1. The Nonlinear Spectral Analysis (NSA) revealed that the nonlinear mechanism in nonlinear finite difference schemes, has a severe impact on the spectral properties of these schemes. First, the nonlinearity leads to an erratic behaviour of the modified wavenumber in the entire wavenumber range $0<\kappa<\kappa_{\max }$, caused by the spurious contributions to the Fourier modes in the entire wavenumber range. Based on the evaluation of the nonlinear schemes for a set of $N_{r}=10^{5}$ synthetic fields, we found that the fluctuations of the modified wavenumber are normally distributed around a mean modified wavenumber. Comparison of the NSA-results with the quasi-linear ADR-results of Pirozzoli [1] show significant differences between both approaches. Indeed, it reveals that the nonlinearity strongly determines the real behaviour of the mean modified wavenumber. Hence, in order to obtain a realistic image about the spectral properties of any nonlinear scheme, the nonlinearity must be accounted for in the analysis. Further, the spreading on the erratic fluctuations, determined by the standard deviation, was found to be significant for upwind schemes as well as for the TVD scheme and the WENO scheme. Indeed, the standard deviation clearly showed that the nonlinearity may induce negative dispersion relations for Fourier modes in the high wavenumber range. Moreover, the nonlinearity may induce even anti-dissipation for modes in the low wavenumber range, in particular for the TVD and the WENO scheme. For the nonlinear DFD scheme, the spreading remained limited in the entire wavenumber range. Despite the central discretization stencil in the DFD scheme, the fluctuations can cause dissipation or anti-dissipation, although in the mean, dissipation is absent.

2. Further, the error on the modified wavenumber indicated that the nonlinear mechanism, responsible for the creation of spurious mode contributions, disturbs the accuracy of all scales, and in particular of the scales in the low-wavenumber range. Indeed, both the theoretical NSA results and numerical results showed that the order of accuracy of the large scales reduces to $1^{\text {st }}$-order and even less. 
3. The nonlinear mechanism is also found to be responsible for the creation of spurious modes behind the cutoff wavenumber of the original synthetic field, i.e. $\kappa_{c}<\kappa<\kappa_{\max }$. This supports the conclusions of Ladeinde et al. [8], who found that ENO and WENO schemes generate numerical turbulence, and Fauconnier et al. [6] who found similar results for the nonlinear DFD scheme. The relative importance of these spurious modes in comparison with the regular modes, depends on the type of discretization. Unless the solution is filtered, these spurious modes interact with all other modes in a real simulation, resulting in aliasing errors that may affect the evolution of the solution. Using the NSA for a single evaluation of the nonlinear discretization for a synthetic field, we found that the aliasing errors of the TVD and the WENO scheme contribute significantly to the total numerical dissipation $( \pm 25 \%)$. For the DFD scheme, the mean aliasing error was zero due to the occurrence of positive and negative contributions to the total kinetic energy.

4. We showed analytically for the linear convection equations, that nonlinear schemes can locally and globally conserve momentum if the nonlinear discretization scheme is written in the divergence formulation. However, we found that nonlinear finite difference discretizations can never conserve the total kinetic energy locally due to the nonlinear mechanism, even if all discretization stencils are central. As a consequence, we investigated the impact of the nonlinearity on the energy balance using the NSA. Again, a significant spreading was found on the spectral distribution of the dissipation rate for the TVD scheme, the WENO scheme and the nonlinear DFD scheme. Nevertheless, in the mean, the TVD and the WENO schemes remain dissipative whereas the DFD scheme lacks dissipation.

We emphasize that this work does not involve any intention to judge the applicability of nonlinear schemes in CFD or CAA, but merely attempts to contribute to a better understanding of nonlinear discretization operators.

\section{Appendix A. Conservative formulation of nonlinear schemes.}

The skew-symmetric formulation of the nonlinear term in Burgers' equation (117) is given by

$$
\text { Skew }=\frac{u}{3} \frac{\delta u}{\delta x}+\frac{1}{3} \frac{\delta u^{2}}{\delta x} .
$$

The skew-symmetric formulation is a combination of the advective and divergence formulations of the nonlinear term. The finite difference discretization of this form is described below for the upwind schemes, the TVD scheme, the $5^{\text {th }}$ -order WENO scheme and the nonlinear DFD scheme.

1. Using $u$ at the intermediate positions $i \pm \frac{1}{2}$, i.e.

$$
u_{i+\frac{1}{2}}=\frac{u_{i}+u_{i+1}}{2} ; \quad u_{i-\frac{1}{2}}=\frac{u_{i}+u_{i-1}}{2},
$$

the $1^{\text {st }}, 2^{\text {nd }}$ and $3^{\text {rd }}$-order upwind discretizations for the skew-symmetric convective term (A.1) are respectively 


$$
\begin{aligned}
\operatorname{Skew}_{i} & =\frac{1}{3} \frac{\left[u_{i}^{+} u_{i}+u_{i}^{-} u_{i+1}\right]-\left[u_{i}^{+} u_{i-1}+u_{i}^{-} u_{i}\right]}{\Delta}+\frac{1}{3} \frac{\left[u_{i+\frac{1}{2}}^{+} u_{i}+u_{i+\frac{1}{2}}^{-} u_{i+1}\right]-\left[u_{i-\frac{1}{2}}^{+} u_{i-1}+u_{i-\frac{1}{2}}^{-} u_{i}\right]}{\Delta} \\
\text { Skew }_{i} & =\frac{1}{3} \frac{\left[u_{i}^{+}\left(3 u_{i}-u_{i-1}\right)+u_{i}^{-}\left(3 u_{i+1}-u_{i+2}\right)\right]-\left[u_{i}^{+}\left(3 u_{i-1}-u_{i-2}\right)+u_{i}^{-}\left(3 u_{i}-u_{i+1}\right)\right]}{2 \Delta} \\
& +\frac{1}{3} \frac{\left[u_{i+\frac{1}{2}}^{+}\left(3 u_{i}-u_{i-1}\right)+u_{i+\frac{1}{2}}^{-}\left(3 u_{i+1}-u_{i+2}\right)\right]-\left[u_{i-\frac{1}{2}}^{+}\left(3 u_{i-1}-u_{i-2}\right)+u_{i-\frac{1}{2}}^{-}\left(3 u_{i}-u_{i+1}\right)\right]}{2 \Delta} \\
\text { Skew }_{i} & =\frac{1}{3} \frac{\left[u_{i}^{+}\left(2 u_{i+1}+5 u_{i}-u_{i-1}\right)+u_{i}^{-}\left(2 u_{i}+5 u_{i+1}-u_{i+2}\right)\right]}{6 \Delta} \\
& -\frac{1}{3} \frac{\left[u_{i}^{+}\left(2 u_{i}+5 u_{i-1}-u_{i-2}\right)+u_{i}^{-}\left(2 u_{i-1}+5 u_{i}-u_{i+1}\right)\right]}{6 \Delta} \\
& +\frac{1}{3} \frac{\left[u_{i+\frac{1}{2}}^{+}\left(2 u_{i+1}+5 u_{i}-u_{i-1}\right)+u_{i+\frac{1}{2}}^{-}\left(2 u_{i}+5 u_{i+1}-u_{i+2}\right)\right]}{6 \Delta} \\
& -\frac{1}{3} \frac{\left[u_{i-\frac{1}{2}}^{+}\left(2 u_{i}+5 u_{i-1}-u_{i-2}\right)+u_{i-\frac{1}{2}}^{-}\left(2 u_{i-1}+5 u_{i}-u_{i+1}\right)\right]}{6 \Delta} .
\end{aligned}
$$

2. Using the same definitions for $u_{i+\frac{1}{2}}, u_{i-\frac{1}{2}}, u^{+}$and $u^{-}$, the TVD discretization of the skew-symmetric nonlinear term is

$$
\begin{aligned}
\text { Skew }_{i} & =\frac{1}{3} \frac{\left[u_{i}^{+}\left(u_{i}+\frac{\psi\left(r_{i}\right)}{2}\left(u_{i+1}-u_{i}\right)\right)+u_{i}^{-}\left(u_{i+1}-\frac{\psi\left(\frac{1}{r_{i+1}}\right)}{2}\left(u_{i+1}-u_{i}\right)\right)\right]}{\Delta} \\
& -\frac{1}{3} \frac{\left[u_{i}^{+}\left(u_{i-1}+\frac{\psi\left(r_{i-1}\right)}{2}\left(u_{i}-u_{i-1}\right)\right)+u_{i}^{-}\left(u_{i}-\frac{\psi\left(\frac{1}{r_{i}}\right)}{2}\left(u_{i}-u_{i-1}\right)\right)\right]}{\Delta} \\
& +\frac{1}{3} \frac{\left[u_{i+\frac{1}{2}}^{+}\left(u_{i}+\frac{\psi\left(r_{i}\right)}{2}\left(u_{i+1}-u_{i}\right)\right)+u_{i+\frac{1}{2}}^{-}\left(u_{i+1}-\frac{\psi\left(\frac{1}{r_{i+1}}\right)}{2}\left(u_{i+1}-u_{i}\right)\right)\right]}{\Delta} \\
& -\frac{1}{3} \frac{\left[u_{i-\frac{1}{2}}^{+}\left(u_{i-1}+\frac{\psi\left(r_{i-1}\right)}{2}\left(u_{i}-u_{i-1}\right)\right)+u_{i-\frac{1}{2}}^{-}\left(u_{i}-\frac{\psi\left(\frac{1}{r_{i}}\right)}{2}\left(u_{i}-u_{i-1}\right)\right)\right]}{\Delta}
\end{aligned}
$$

in which the ratio of consecutive difference is still given by relation (9).

3. Adopting following expressions,

$$
\begin{array}{rlrl}
u^{+} & =\frac{1}{2}(u+\theta u), & \theta=1 \\
u^{-} & =\frac{1}{2}(u-\theta u), \quad \theta=1 \\
\left(u^{2}\right)^{+} & =\frac{1}{2}\left(u^{2}+\theta u\right), \quad \theta=\max (|u|) \\
\left(u^{2}\right)^{-} & =\frac{1}{2}\left(u^{2}-\theta u\right), \quad \theta=\max (|u|),
\end{array}
$$


the $5^{\text {th }}$-order WENO scheme, applied to the skew-symmetric nonlinear term, is

$$
\operatorname{Skew}_{i}=\frac{u_{i}}{3} \frac{\left(u_{i+\frac{1}{2}}^{+}+u_{i+\frac{1}{2}}^{-}\right)-\left(u_{i-\frac{1}{2}}^{+}+u_{i-\frac{1}{2}}^{-}\right)}{\Delta x}+\frac{1}{3} \frac{\left(\left(u^{2}\right)_{i+\frac{1}{2}}^{+}+\left(u^{2}\right)_{i+\frac{1}{2}}^{-}\right)-\left(\left(u^{2}\right)_{i-\frac{1}{2}}^{+}+\left(u^{2}\right)_{i-\frac{1}{2}}^{-}\right)}{\Delta x} .
$$

The expressions for the positive and negative contributions of $u$ and $u^{2}$ at the intermediate positions, are identical to those defined in section 2 .

4. Finally, the central nonlinear DFD scheme, for the discretization of the skew-symmetric term, is

$$
\begin{aligned}
\text { Skew }_{i} & =\frac{u_{i}}{3} \frac{\left[u_{i+1}+c_{i+1}\left(u_{i+2}-2 u_{i+1}+u_{i}\right)\right]-\left[u_{i-1}+c_{i-1}\left(u_{i}-2 u_{i-1}+u_{i-2}\right)\right]}{2 \Delta} \\
& +\frac{1}{3} \frac{\left[u_{i+1}^{2}+c_{i+1}\left(u_{i+2}^{2}-2 u_{i+1}^{2}+u_{i}^{2}\right)\right]-\left[u_{i-1}^{2}+c_{i-1}\left(u_{i}^{2}-2 u_{i-1}^{2}+u_{i-2}^{2}\right)\right]}{2 \Delta} .
\end{aligned}
$$

The dynamic coefficient $c$ is still determined as

$$
c_{i}=-\frac{1}{6} \frac{1}{1+f \max \left(\min \left(\frac{u_{i+2}-4 u_{i+1}+6 u_{i}-4 u_{i-1}+u_{i-2}}{u_{i+1}-2 u_{i}+u_{i-1}}, 0\right),-3\right)} .
$$

\section{References}

[1] S. Pirozzoli, Short note: On the spectral properties of shock-capturing schemes, J. Comput. Phys. 219 (2) (2006) $489-497$. doi:http://dx.doi.org/10.1016/j.jcp.2006.07.009.

[2] R. Courant, E. Isaacson, M. Rees, On the solution of nonlinear hyperbolic differential equations by finite differences, Comm. Pure Appl. Math. 5 (1952) 243-255.

[3] A. Harten, High resolution schemes for hyperbolic conservation laws, Journal of Computational Physics 49 (3) (1983) 357 - 393. doi:DOI:10.1016/0021-9991(83)90136-5.

URL http://www.sciencedirect.com/science/article/B6WHY-4DD1XP3-1PC/2/7139ec41e5bce99e04612c431428ead2

[4] W. Chi-Shu, Essentially non-oscillatory and weighted essentially non-oscillatory schemes for hyperbolic conservation laws, Tech. rep. (1997).

[5] A. Harten, B. Engquist, S. Osher, S. R. Chakravarthy, Uniformly high order accurate essentially non-oscillatory schemes, iii, Journal of Computational Physics 71 (2) (1987) 231 - 303. doi:DOI : 10. 1016/0021-9991(87)90031-3.

URL http://www.sciencedirect.com/science/article/B6WHY-4DD1T0N-J9/2/e95d1dd8f52261439068df272f 21f4dd

[6] D. Fauconnier, C. De Langhe, E. Dick, A family of dynamic finite difference schemes for large-eddy simulation, Journal of Computational Physics 228 (6) (2009) 1830 - 1861. doi:DOI:10.1016/j.jcp.2008.11.014.

URL http://www. sciencedirect.com/science/article/B6WHY-4V166HB-1/2/6560b8e8b9a39b011a31db32f096df63

[7] D. Fauconnier, C. De Langhe, E. Dick, Construction of explicit and implicit dynamic finite difference schemes and application to the large-eddy simulation of the taylor-green vortex, Journal of Computational Physics 228 (21) (2009) 8053 - 8084. doi:DOI: $10.1016 / j \cdot j c p .2009 .07 .028$.

URL http://www.sciencedirect.com/science/article/B6WHY-4WXBMGY-2/2/1de78ad936a075a15c03c018bb6e3a5c

[8] F. Ladeinde, X. Cai, M. R. Visbal, D. V. Gaitonde, Turbulence spectra characteristics of high order schemes for direct and large eddy simulation, Appl. Numer. Math. 36 (4) (2001) 447-474. doi:http://dx.doi .org/10.1016/S0168-9274(00) 00019-2.

[9] C. Bogey, N. de Cacqueray, C. Bailly, A shock-capturing methodology based on adaptative spatial filtering for high-order non-linear computations, Journal of Computational Physics 228 (5) (2009) 1447 - 1465. doi:DOI:10.1016/j.jcp. 2008.10.042.

URL http://www. sciencedirect.com/science/article/B6WHY-4TY49KC-3/2/00903dc19c4c42883f2488ca2016e766

[10] S. R. Chakravarthy, S. Osher, High resolution applications of the osher upwind scheme for the euler equations, in: Sixth AIAA CFD Conference, AIAA, Danvers, MA, USA, 1983, p. 363.

[11] R. Wang, R. J. Spiteri, Linear instability of the fifth-order weno method, SIAM J. Numer. Anal. 45 (2007) $1871-1901$. doi:10.1137/050637868.

URL http://portal . acm. org/citation. cfm?id=1330026.1330032

[12] S. A. Orszag, On the elimination of aliasing in finite-difference schemes by filtering high-wavenumber components, Journal of the Atmospheric sciences 28 (1971) 1074.

[13] J. G. Wissink, On unconditional conservation of kinetic energy by finite-difference discretizations of the linear and non-linear convection equation, Computers \& Fluids 33 (2) (2004) 315 - 343. doi : DOI : 10.1016/S0045-7930 (03)00057-4. URL http://www. sciencedirect.com/science/article/B6V26-497YSXC-4/2/3450300a0f7106c72ff481fb058d4fc1

[14] Y. Morinishi, T. S. Lund, O. V. Vasilyev, P. Moin, Fully conservative higher order finite difference schemes for incompressible flow, J. Comput. Phys. 143 (1) (1998) 90-124. 
[15] Y. Morinishi, Skew-symmetric form of convective terms and fully conservative finite difference schemes for variable density low-mach number flows, J. Comput. Phys. 229 (2010) 276-300. doi:10.1016/j . jcp. 2009.09. 021.

URL http: //portal.acm.org/citation. cfm?id=1655423.1655669

[16] J. C. Kok, A high-order low-dispersion symmetry-preserving finite-volume method for compressible flow on curvilinear grids, J. Comput. Phys. 228 (2009) 6811-6832. doi:10.1016/j.jcp.2009.06.015.

URL http://portal.acm.org/citation. cfm?id=1618877.1619057

[17] P. K. Subbareddy, G. V. Candler, A fully discrete, kinetic energy consistent finite-volume scheme for compressible flows, J. Comput. Phys. 228 (2009) 1347-1364. doi:10.1016/j.jcp.2008.10.026.

URL http://portal.acm.org/citation.cfm?id=1497640.1497955

[18] S. Pirozzoli, Generalized conservative approximations of split convective derivative operators, J. Comput. Phys. 229 (2010) $7180-7190$. doi:http://dx.doi.org/10.1016/j.jcp.2010.06.006

URL http://dx.doi.org/10.1016/j.jcp.2010.06.006

[19] H. Aref, P. K. Daripa, Note on finite difference approximations to burgers' equation, SIAM Journal on Scientific and Statistical Computing 5 (4) (1984) 856-864. doi:10.1137/0905060.

URL http://link. aip.org/link/?SCE/5/856/1 